\title{
Combination regimens with PD-1/PD-L1 immune checkpoint inhibitors for gastrointestinal malignancies
}

\author{
Dongxu Wang ${ }^{1 \dagger}$, Jianzhen Lin ${ }^{1 \dagger}$, Xu Yang ${ }^{1 \dagger}$, Junyu Long ${ }^{1}$, Yi Bai ${ }^{1}$, Xiaobo Yang ${ }^{1}$, Yilei Mao ${ }^{1}$, Xinting Sang ${ }^{1}$, \\ Samuel Seery ${ }^{2}$ and Haitao Zhao ${ }^{1 *}$ (i)
}

\begin{abstract}
Gastrointestinal (Gl) malignant neoplasms have a high global incidence and treatment prospects for patients with advanced Gl tumors are dismal. PD-1/PD-L1 inhibitors emerged as a frontline treatment for several types of cancer. However, the shortcomings of PD-1/PD-L1 inhibitors have been observed, including low objective response rates and acquired tumor resistance, especially in patients receiving PD-1/PD-L1 inhibitors as a single treatment. Accumulating evidence from clinical trials increasingly suggests that combined immunotherapies enhance therapeutic responses in patients with malignances, especially for Gl tumors which have a complex matrix, and significant molecular and immunological differences. Preclinical and clinical studies suggest there are advantages to combined immunological regimens, which represents the next logical step in this field, although further research is necessary. This literature review explores the current limitations of monotherapies, before critically discussing the rationale behind combination regimens. Then, we provide a summary of the clinical applications for gastrointestinal cancers.
\end{abstract}

Keywords: Gastrointestinal malignancies, PD-1/PD-L1 blockade, Immune checkpoint inhibitor, Combination immunotherapy, Clinical application, Rationale, Clinical trial

\section{Background}

Gastrointestinal (GI) neoplasms threaten human health and account for approximately $35 \%$ of all cancer-related mortalities among common malignancies [1]. Typically, patients are diagnosed accidentally with latent, unspecific symptoms reducing the already limited number of possible interventions. Surgical resection can be curative; however, the majority of patients are diagnosed in the advanced stages of this condition, therefore the opportunity for a radical cure is lost. The prevalence and impact of this insidious disease as well as limited treatment options necessitates the systematic search for innovative evidence-based treatments.

Advances in our understanding of immune-system/ tumor interactions have led researchers to uncover new

\footnotetext{
* Correspondence: ZhaoHT@pumch.cn

${ }^{\dagger}$ Dongxu Wang, Jianzhen Lin, and Xu Yang are equal contributors.

${ }^{1}$ Department of Liver Surgery, Peking Union Medical College Hospital, Chinese Academy of Medical Sciences and Peking Union Medical College, Beijing, China

Full list of author information is available at the end of the article
}

diagnostic pathways which may result in earlier identification. Also, several immunotherapies for the treatment of GI tumors have recently emerged. Among these new interventions, immune checkpoint inhibitor therapies are perhaps the most promising strategy [2]. Indeed, the findings from many clinical trials suggest that immunological checkpoint blockade therapies may be effective for various types of tumor, with durable responses and manageable toxicity, regardless of pathologic grade [3]. For those with GI tumors, blocking programmed cell death protein-1 (PD-1/CD279) or the ligand PD-L1 is also effective in approximately $20-40 \%$ patients. Due to such outcomes and with this moderate success, PD-1/ PD-L1 blockades have been approved by the FDA for advanced colorectal, gastric, and liver cancers.

In contrast to other tumors such as lung cancer and breast cancer, GI tumors have mesenchymal traits which hinder the infiltration of immune cells thereby crippling the antitumor response [4]. Likewise, the immunotherapeutic effects upon digestive tract tumors vary substantially which is perhaps due to different molecular and 
immunological characteristics. As such, several researchers have called for GI tumors to be reclassified based upon molecular type rather than around anatomical systems and histological features only [5]. Despite this call for change, high mortality rates associated with these malignancies continues to drive clinical research in this field. Several phase I-III trials focusing on immunotherapies for GI tumors have found what can only be described as unsatisfactory objective response rates (ORR), ranging between 10 and $25 \%$ [6]. In addition, problems such as drug resistance and the side effects of anti-PD-1/PD-L1 treatments remain challenging [7]. So, while this growing body of evidence suggests that target-driven treatment strategies are essential, there is a paucity of research from which to design new interventions.

Presently, the logical next step appears to be combining immunotherapies with antitumor drugs and some progress has been made in preclinical and clinical studies which suggest that combined immunotherapies may increase benefit. However, this is a relatively new field of study hence effort should be made to embed research systematicity using secondary literature. As such, this study focuses on reviewing the current limitations of immune checkpoint blockade monotherapies and to critically discuss the rationale behind combination strategies based on the PD-1/PD-L1 blockade. The aim is to provide researchers and practitioners with a summary of the clinical applications of combination therapies for patients with upper and lower GI tumors and to explore the arguments around combination immunotherapies.

\section{PD-1/PD-L1 pathway blockade: current limitations in clinical treatment}

The immune checkpoint pathway composed of PD-1/ CD279 and the related ligand PD-L1 evade immune surveillance by upregulating the expression in tumor cells during the progress of $\mathrm{T}$ cell-mediated immune killing. Substantial evidence from preclinical models indicates that blocking PD-1/PD-L1 interactions can enhance immune normalization and reinforce anticancer responses $[8,9]$. As early as 2003 , Chen et al. found that using the B7 homolog 1 (B7-H1) blocking antibody combined with $\mathrm{T}$ cell transfusion cured approximately $60 \%$ of the 24 mice with squamous cell carcinomas in the head and neck. Without the transfusion of $\mathrm{T}$ cells, only one of five mice treated with $\mathrm{B} 7-\mathrm{H} 1$ blockade had prolonged survival; however, this was not considered a statistically significant improvement compared with the control group [10].

In 2012, a phase I clinical trial investigating the efficacy of pembrolizumab for patients with advanced tumors found that the objective response rate (ORR) for patients with advanced non-small cell lung cancer (NSCLC), malignant melanoma, and advanced renal carcinoma was $18 \%, 28 \%$, and $27 \%$, respectively, and the adverse event profile does not appear to preclude its use [11]. Similarly, a longitudinal study focusing on pretreated advanced NSCLC, involving 129 patients found a 16\% five-year survival rate. While this study contained a larger number of participants which adds precision, pretreatments were not standardized. Nevertheless, this study suggests that PD-1 blockading may prolong therapeutic durability [12]. This evidence of antitumor activation and the antibodies targeting the capabilities of PD-1/PD-L1 convinced the FDA to officially approve five inhibitors. The preliminary indications were that these inhibitors could be administered for several different types of tumor, including microsatellite instability-high (MSI-H) solid tumors.

The main advantages of PD-1/PD-L1 inhibitors are effect persistence (i.e., durability) and the broad-spectrum effects of these agents. However, the noticeable deficiency of PD-1/PD-L1 blockades is inconsistency across a homogeneous study population with similar tumor characteristics [13]. The exception to this can be observed in tumors with specific genetic changes, such as MSI-H, deficient mismatch repair (dMMR), and high tumor mutational burden (TMB). A review of the status and perspectives of translational biomarkers found the ORR is only $15-25 \%$ for unscreened solid tumors and even lower for some tumors, such as colorectal and pancreatic cancer [14] which suggests the causal factor for this relatively low response rate might be attributed to tumor heterogeneity, genetic variation among individuals, and perhaps structural differences between blockades [15]. Although, studies have also found that the development and evolution within a tumor itself can lead to a decreased efficacy of the PD-1 blockade. This may be due to genetic alterations within DNA encoding immunogenic signaling pathway proteins, a lack of sufficient mutation-associated neoantigens (MANAs) in the presence of an immunosuppressive tumor microenvironment, and/or the unmasking of immunogenicity by immune checkpoint inhibitors (ICPIs) to induce an enhanced antitumor response [16].

As well as increasing antitumour activity, PD-1/PD-L1 blockade treatments may also cause certain inflammatory side effects in some patients which are referred to as immune-related adverse events (irAEs) $[17,18]$. Essentially, these immunotherapies unbalance the immune system, generating dysimmune toxicities which potentially effect any tissue. However, a systematic review of the side effects of the PD-1/PD-L1 blockade suggests irAEs can be widespread but are more likely to involve the GI tract, endocrine glands, and skin [19-21]. Compared to the side effects of chemotherapy, immunotherapeutic side effects appear more diverse, random, and differential but primarily organ-based manifestations [17]. Some studies indicate that these irAEs may be closely related to the expression and distribution of PD-L1 and PD-L2 [22-24] which suggests while irAEs 
may be heterogeneous in nature, they may be tolerable and most associated side effects are treatable. However, there are potentially serious adverse reactions, such as myocarditis which can cause death. A substantial increase in the number of deaths associated with immune checkpoint inhibitors has been observed, although this may be attributed to increased use and raised awareness of this clinical entity [25]. Conversely, some irAE studies have found improved immune responses in patients which suggest that these might also be used to predict treatment efficacy [26].

The efficacy of PD-1/PD-L1 blockades can be lasting for some patients, although tumor development remains a constant threat even under continuous therapy [27]. In a screening evaluation of PD-1 for the treatment of malignant melanoma, 48 cases were found to have significantly reduced tumor size or stable progression. However, in approximately half of those participants, tumors initially shrank before increasing in size directly after receiving this intervention [28]. This suggests that this treatment may have had little or no effect overall due to immunotherapeutic resistance. At present, the possible mechanisms of acquired immunotherapy resistance appear to include loss-of-function mutations in beta-2-microglobulin (B2M) and Janus kinases (JAK1 and JAK2) [29].

A study of two fully immunocompetent mouse models focusing on lung adenocarcinoma indicate that the $\mathrm{T}$ cell immunoglobulin mucin-3 (TIM-3) was upregulated in tumors resistant to PD-1 blockade, and a survival advantage was found with the addition of a TIM-3 blocking antibody following failure of the PD-1 blockade. This suggests that there may be a targetable biomarker associated with adaptive resistance to PD-1 blockades [30]. Early clinical investigations have also found some patients with complete remission after treatment with PD-1/PD-L1 blockades, relapse. Although, data related to this phenomenon is limited, it does suggest a lack of therapeutic durability in humans which is supported by basic medical evidence.

Adding to the aforementioned side effects and drug resistance after immunotherapy, studies indicate that a small number of patients on PD-1 blockades will experience hyper-progression [31-33]. The Ferrara study, which included 242 patients, found that tumor growth rates increased by more than $50 \%$ in $16 \%$ of patients $(n$ $=40$ ) after receiving the PD-1 antibody. This finding meets the criteria for hyper-progression; however, this study lacked a control group and determining tumor progression causality was not possible [34]. To explore this phenomenon in more detail, Singavi et al. conducted an analysis of somatic alterations looking into the biomarkers for hyper-progression and found that copy number alterations in murine double minute $2 / 4$ (MDM2/MDM4), the epidermal growth factor receptor
(EGFR), and several genes located on 11q13 are associated with hyper-progression. The role of these somatic alterations as putative predictive biomarkers for hyperprogression requires further investigation with larger samples [35].

Identifying biomarkers is crucial as these might support both treatment efficacy and AE predictions in patients receiving immunological checkpoint therapy [36]. Biomarkers such as $\mathrm{AMMR}$ and MSI, TMB and blood TMB, HLA diversity and PD-L1 expression have been explored. While stable predictors are not, presently available, different regions of the body develop different types of tumor, therefore antibodies used for detecting PD-L1 expression may be highly specific to one region while insensitive to level of expression, and vice versa. Furthermore, the activation effect of subsequent treatments is likely to change PD-L1 expression [37], a factor which is currently adopted in clinical trials to predict immunological efficacy [38]. While TMB, dMMR, and MSI positively correlate with the efficacy of PD-1, they are not widely used due to the limitations of these detection techniques [39]. In summary, our knowledge of these biomarkers is far from complete therefore cannot be used as guidelines for precision immunotherapy. Alternative predictive markers are currently in the early exploratory phase [40, 41].

\section{Combination immunotherapy: future steps for effective immunotherapy}

Rationale behind combined immunotherapies based on PD-1/L1 blockade

The limitations of monotherapy with PD-1/PD-L1 blockades and the lack of promising alternatives has made it necessary to seek combination treatment methods which can activate antitumor immunity and enhance treatment efficacy. Studies on the interactions between the immune system and tumors indicate the cancer-immunity cycle primarily involves the following steps. Firstly, tumor-antigens are released to be processed by anti-presenting cells (APCs) which migrate to lymphoid organs. Then, $\mathrm{T}$ cells are activated and fine-tuned through co-stimulation and co-inhibitory signals which regulate naive tumor-specific $\mathrm{T}$ cells, encouraging them toward the tumor tissues and to become effector T cells in lymphoid organs. The regression of tumor-specific effector $\mathrm{T}$ cells which occurs from lymphoid organs into the peripheral blood is known as adoptive $\mathrm{T}$ cell transfer and trafficking. The final stage occurs when cytotoxic $\mathrm{T}$ cells attack tumor cells leading to tumor lysis as well as encouraging tumor-specific memory T cells $[9,42,43]$. From activation to cytotoxicity, $\mathrm{T}$ cell-led cellular immune regulation mainly progress through the final three steps described $[43,44]$.

Any abnormality in processing $\mathrm{T}$ cell immune clearance can lead to a decrease or even the disappearance of antitumor effects. Therefore, tumor tissues can escape a 
$\mathrm{T}$ cell attack through three regulatory mechanisms. There is the adaptive immune system and the natural immune systems inhibition of cell recruitment, such as the recruitment of regulatory $\mathrm{T}$ cells (Tregs) and myeloid-derived suppressor cells (MDSCs). Tumors can also escape variant selection and tumor-associated antigen expression loss, as well as co-stimulatory molecule downregulation and immunosuppressive factor secretion [45]. Based upon this current understanding, a hypothesis emerged that antitumor drugs which potentially enhance $T$ cell immunity and interfere with tumor immune responses can be combined with a PD-1 blockade thereby exerting a synergistic antitumor effect. However, caution must be given because this is a generalized theory which may only apply to specific types of carcinoma.

GI neoplasms have a complex matrix microenvironment which includes a variety of cell types, extracellular matrices, and metabolic mediators $[4,46]$. Each of these components can become obstacles for cytotoxic $\mathrm{T}$ lymphocytes (CTLs) which may enable tumor cells to evade attack by antitumor drugs. Different types of tumor cells have different levels of immunogenicity, related to cell surface antigens which activate immune cells. This variability creates different levels of tumor resistance and varying immune responses. As such, tumors can be categorized into different immunity-related phenotypes (i.e., immune-desert phenotype, immune-excluded phenotype or immune inflammatory phenotype) [47]. Within the immune desert phenotype, the tumor micro environment lacks immune-effector $\mathrm{T}$ cells because immunogenicity functions are inhibited. The result of which is ineffective $T$ cell priming or activation. Although, sufficient effector $\mathrm{T}$ cells exist near the tumor in the immune-excluded phenotype, $\mathrm{T}$ cells appear to be blocked between the stroma and the tumor parenchyma. Therefore, $\mathrm{T}$ cells cannot penetrate to attack tumors. Both immune-desert and immune-excluded phenotype tumors are considered cold tumors, which are associated with low responsiveness [48].

Unlike immune-desert or immune-excluded phenotypes, immune inflammatory phenotypes are considered hot tumors which suggest they may be highly sensitive and responsive to treatment with a PD-1/PD-L1 blockade. One of the purposes of combination immunotherapies is to convert cold tumors into hot tumors by altering the tumor micro environment, thereby enhancing immune efficiency $[48,49]$. Generally, tumors contain a large number of CD4/CD8 ${ }^{+} \mathrm{T}$ cells, APCs, and monocytes [47]. As such, combing PD-1/PD-L1 blockade therapies can be further rationalized because it is hoped this will enhance the presentation of tumor antigens, including the antigen-presenting function of antigen-presenting cells. Combining PD-1/PD-L1 may also enhance immune auxiliary functions thereby increasing the infiltration of $\mathrm{T}$ cells and the activity of CTLs in tumor tissues. Also, combination therapies may enhance tumor cell immunogenicity while reducing the efficacy of immunosuppressive molecules such as indoleamine 2,3-dioxygenase (IDO), C-X-C Motif chemokine receptor 2 (CXCR2), lymphocyte-activation gene 3 (LAG-3), phosphoinositide 3-kinase (P13K), for example $[48,50]$.

Traditional treatment methods, including combined radiotherapy with chemical interventions, have had limited success but can kill tumor cells which may lead to the release of tumor-specific antigens, thereby initiating immune clearance [51]. Targeted therapies focusing on tumor-specific gene mutation show promise and therefore are likely candidates for further investigation. In addition, evidence suggests PD-1/PD-L1 blockades can be combined directly with other immune checkpoint inhibitors (ICPIs), including some immunosuppressive small molecule blockades, having compatible, and theoretically complementary modalities. This emergent approach is commonly referred to as double immunotherapy and the mechanisms of multiple combined treatments are summarized below in Fig. 1.

The purpose of exploring combined interventions is to combine specific antitumor modalities to enhance therapeutic effects. However, this must be an evidence-based investigation to reduce both risk and harm. In contrast to immune monotherapy, several studies have shown that combined immunological regimens increase the incidences of all grade irAEs, especially for double immunotherapy [52]. The severity of adverse events in combination therapies also differs from monotherapies. For example, a study of nivolumab combined with ipilimumab compare to monotherapy for untreated melanoma found grade 3 or 4 irAEs occur in $55 \%$ of those in the combination cohort, while in the nivolumab cohort and ipilimumab cohort this was only $16.3 \%$ and $27.3 \%$, respectively [53]. Of note, in contrast to the mechanisms of traditional tumor agents which target the tumor in situ, immunotherapies exert an antitumor effect through the activation of the immune system, creating different adverse events profiles. Under these circumstances, immunotherapies combined with traditional antitumor agents may result in a more complex set of adverse events $[54,55]$. Therefore, combined regimens for immunotherapy present specific challenges that must be considered with respect to the evaluation of treatment related adverse events.

\section{Approaches and mechanisms for combining PD-1/PD-L1 with antitumor therapies PD-1/PD-L1 blockades combined with other T cell checkpoint inhibitors}

There are two critical steps of $\mathrm{T}$ cell activation which play an essential role in immune homeostasis. These 


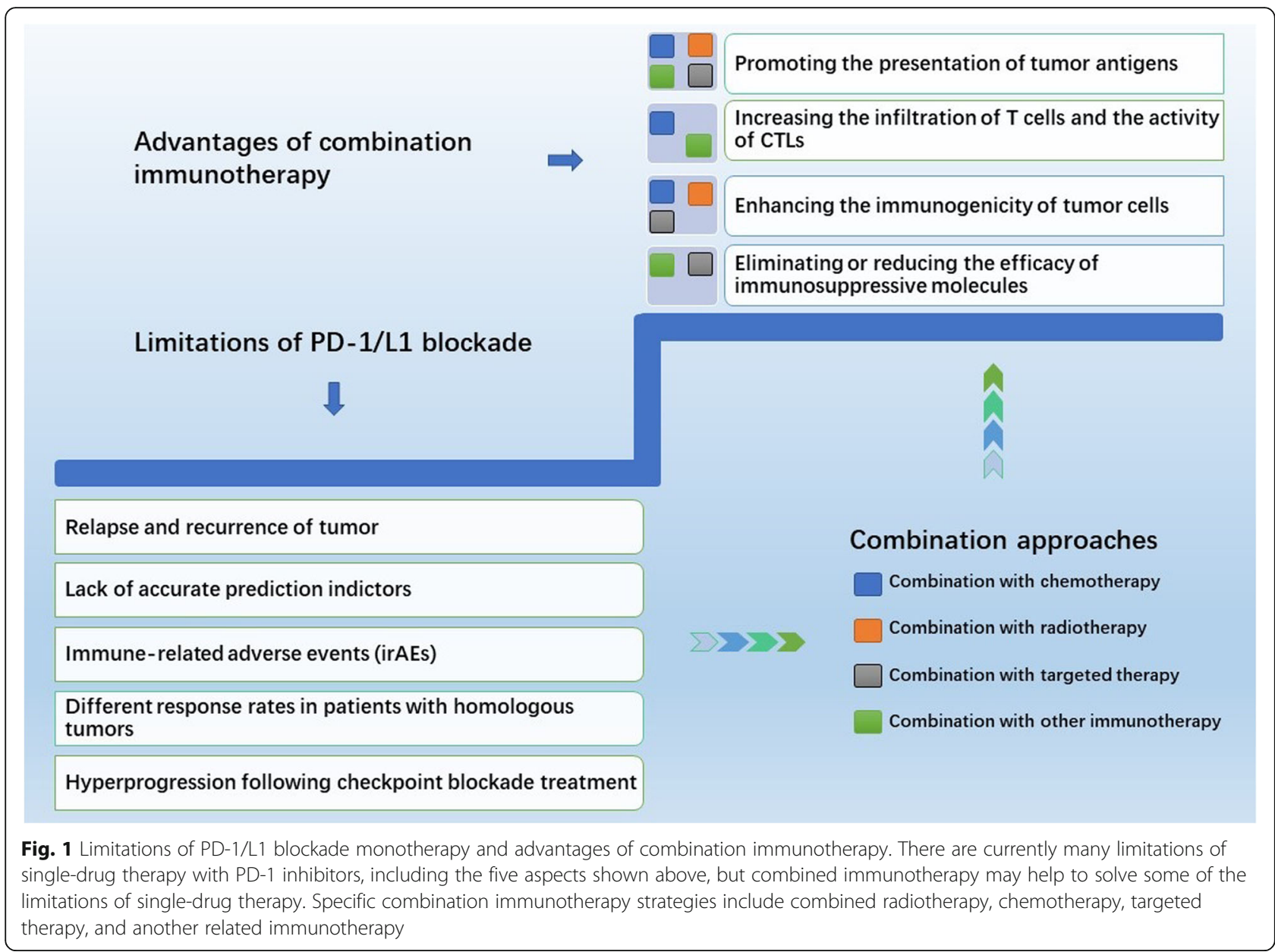

steps involve multiple immune checkpoint pathways within the cancer-immunity cycle. Research suggests that several ICPIs may enhance the activity of cytotoxic $T$ cells by antagonizing regulatory pathways which inhibit $\mathrm{T}$ cell functions [56]. Similarities and differences in checkpoint pathway mechanisms may be the reason single checkpoint inhibitors do not hold the desired antitumor effect. Combined immunotherapy targeting different immune checkpoints may then increase response rates.

Many immunological checkpoint combinations have been administered both in research and practice, including PD-1 combined with co-inhibitory factors, such as LAG-3, CTLA-4, and TIM-3. Several co-stimulatory factors such as tumor necrosis factor receptor super family member 4 (TNFRSF4), glucocorticoid-induced TNFR family related gene (GITR), and CD137 have also been investigated $[57,58]$. PD-1/PD-L1 combined with CTLA-4 is currently one of the most commonly used immunological checkpoint combinations, and has been approved by the FDA for use in advanced renal carcinoma and melanoma. The mechanisms by which these agents affect immune function are subtly different and so necessary research is ongoing.
Presently, research indicates CTLA4 blockades act within lymph nodes, whereas PD-1/PD-L1 blockades act primarily in tumor tissues [59]. Basic research has demonstrated that after $\mathrm{CD} 8^{+} \mathrm{T}$ cells are removed, inhibiting the PD-1/PD-L1 pathways which fail to initiate tumor killing effects. On the other hand, CTLA-4 blockades also inhibit the B7-CTLA-4 pathway, which can initiate $\mathrm{CD}^{+} \mathrm{T}$ cell proliferation in lymph nodes and increase the infiltration of CTLs into tumor tissues [60]. Additionally, CTLA-4 antagonists may impede tumor inhibition capabilities of Treg cells. Therefore, in tumors with less $\mathrm{T}$ cell infiltration, this combination may have complementary action and clinical research supports this theory, indicating that this combination yields a higher level of treatment efficacy than either agent administered independently [60].

In one study of patients suffering advanced melanoma, the median overall survival (mOS) was surpassed with nivolumab and ipilimumab combined at the 36-month follow up point. Administered separately, the nivolumab and ipilimumab groups resulted in a 37.6 and 19.9 month mOS, respectively [61], indicating this combination does increase longevity for those suffering advanced melanoma. 
Similarly, in patients with advanced renal cell carcinoma, a study of a combination of first-line targeted drugs revealed a $42 \%$ ORR with nivolumab plus ipilimumab compared to $27 \%$ with sunitinib. This study found prolonged longevity where the mOS surpassed the 26 month baseline established with a sunitinib monotherapy [62]. Unfortunately, patients with negative PD-L1 expression are generally considered less likely to respond well to anti-PD-1 monotherapies, therefore may benefit from a combination.

Interestingly, the CheckMate-227 study which compared chemotherapy alone with double immunotherapy found that double immunotherapy can improve mPFS as well as ORR in patients suffering lung cancer, irrespective of PD-L1 expression. Overall, there was a $45.3 \%$ ORR with a corresponding 7.2 mPFS in those whom received nivolumab plus ipilimumab. This finding was in stark contrast to $26.9 \% \mathrm{mPFS}$ and 5.5 month mOS found in those whom had received chemotherapy alone [63]. While these initial findings show promise, supporting evidence with which to generate systematic reviews or meta-analyses focusing on this field is sparse. Having said that, this evidence should be taken indicatively and should drive further research in this area.

\section{PD-1/PD-L1 blockades combined with tumor immunotherapeutic small molecules}

$\mathrm{T}$ cell chemokines, cell chemokine ligand 5 (CCL5), and C-X-C motif chemokine 10 (CXCL10) are associated with a better responses to immunotherapy [64]. The use of small molecular bioeffectors, such as histone deacetylase (HDAC) inhibitors which enhance the expression of $\mathrm{T}$ cell chemokines, may augment response rates to PD-1 blocking immunotherapy [65]. These small molecules can be classified into enzyme inhibitors (i.e., IDO and ARG1), chemokines and their receptors (i.e., the CXCR family), antigen-activated immune response classes (TLRs), signal transduction classes (PI3K- $\gamma$ and BRAF), metabolites, cytokines, and other classes (e.g., COX2) [66]. Most small molecules develop during the preclinical stage; however, some small molecules enter the clinical stage, especially enzyme inhibitors and signal transduction factors. The guiding principle of the synergistic effect for combining small molecule drugs with ICPIs is that this may enhance tumor immunogenicity, which may in turn may enhance the efficacy of immuno-oncological (IO) treatments.

For instance, Indoleamine 2,3-dioxygenase 1 (IDO-1) which catalyzes the decomposition of tryptophan into kynurenic acid, may prevent CTLs from attacking cancer cells while upregulating Treg immunosuppression [67]. Evidence however remains conflicting, with early phase clinical trials suggesting that approximately $58 \%$ of melanoma patients $(n=19)$ receiving IDO inhibitors combined with PD-1 inhibitors achieved a complete response (CR) of $26 \%$ and a $32 \%$ partial response (PR) [68]. Likewise, in the ECHO-204 phase I/II study which combined an IDO inhibitor with nivolumab also yielded promising results in patients with melanomas with ORR and CR rates of $63 \%$ and $5 \%$, respectively. The overall effect size was both enhanced and considered significant when compared with pharmacological interventions alone [69]. However, a recent phase III study suggests that perhaps this effect does not transpose and combining IDO inhibitors with pembrolizumab did not result in a significant, longer PFS when compared with placebo plus pembrolizumab (median 4.7 versus 4.9 months). The PFS rate at 12 months was $37 \%$ in both groups which suggests that there is no improvement. Unfortunately, combining these interventions also manifested in an increased number of side effects compared with PD-1 inhibitors alone [70]. Therefore, a full understanding of the mechanisms of small molecule drugs combined with ICPIs garnered through basic research and phase I/II trials is necessary before large phase III trials are commenced in this area.

\section{PD-1 blockades combined with targeted therapy}

Increasing attention is being given to targeted therapies because the identification of actionable oncogenic driver alterations has improved and we are gaining a deeper understanding of the microenvironments in which tumor develop. Monoclonal antibodies (McAbs) which target tumors mainly include drugs that target tumor-driving genes, inhibit protein kinase complexes by targeting the fusion mutation of EGFR, ALK, etc., or drugs which target angiogenesis (e.g., axitinib or sorafenib). Currently, the efficacy of targeted drugs is limited due to the development of acquired resistance initiated by different molecular mechanisms. However, this can be partially offset considering the durability of PD-1/PD-L1 inhibitors, which may exert a synergistic antitumor effect.

Neoantigens released through the lethal effects of targeted agents may actually strengthen the antitumor immune response [71]. In a melanoma mouse model, dabrafenib significantly increased the infiltration of $\mathrm{CD}^{+} \mathrm{T}$ cells, and trametinib in BRAF wild-type tumor cells appears to upregulate human leukocyte antigen (HLA) molecule expression while downregulating certain immunosuppressive factors such as PD-L1, IL1, IL8, CD73, and vascular endothelial growth factor A (VEGFA) [72]. Anti-angiogenesis drugs may normalize abnormal tumor blood vessels, thereby increasing the infiltration of immunocytes and exerting the anticipated synergistic antitumor effects of immuno-targeted therapy [73]. In addition, antiangiogenic treatment may ameliorate tumor hypoxia and transform the immunosuppressive tumor microenvironment into an immune-enhanced tumor microenvironment [74, 75], although clinical studies are required. 
Thus far, a number of clinical trials have investigated PD-1 blockade combined with antiangiogenic drugs, including combinations with lenvatinib, cabozantinib, bevacizumab, and axitinib. The results of PD-1 blockade combined with lenvatinib suggest that there may be a benefit for patient suffering advanced renal carcinoma (63\% ORR). However, this was a relatively small study $(n=30)$, therefore findings can be only tentatively generalized. In a similar study conducted involving 23 patients suffering endometrial carcinoma, researchers found a $50 \%$ ORR $[76,77]$ which might be considered promising, although not enough is known about the impact of demographic differences or lifestyles. Therefore, while promising, these studies should only be used to initiate larger studies, designed with more comprehensive data collection methods.

Taken together, these studies provide a small and incomplete evidence base for combining targeted drugs with ICPIs. At present, not enough is known about appropriate doses, time sequencing, or individuals which may improve patient prognosis. So, while the FDA has reported this combination as a "breakthrough" in the treatment of advanced renal cell carcinoma, caution must be given. Further, large-scale studies are required before such broad generalizations are presented publicly. Having said that, this is an area which does show promise and is the foundation of an emerging evidence base which should incorporate a focus on dose optimization, sequencing treatments, and demographic differences in order to maximum individual benefit.

\section{PD-1 blockades combined with radiotherapy}

There is a dual effect of radiotherapy on the immune system. On the one hand, radiotherapy inhibits immunity and promotes tumorigenesis. On the other hand, radiotherapy promotes tumor immunogenicity and apoptosis which enhances CD8 T cell tumor-infiltration while stimulating a systemic immune response [78]. Preclinical studies have shown that localized radiotherapy can promote the release of tumor-associated antigens, recruiting immune cells and change the tumor microenvironment which in turn promotes the antitumor immune response [79]. Adding a PD-1 inhibitor after radiotherapy has been administered and might manifest in a prolonged immune memory as has been observed in situ tumor vaccines [80]. Therefore, the role of radiotherapy as a treatment is evolving into perhaps a more powerful adjuvant for immunotherapy.

Radiotherapy can reduce MDSCs developing within the tumor microenvironment, producing new tumor antigens and potentially enhancing antigen presentation. Radiotherapy functions by destroying the tumor matrix and for a short period many antigens are released. These antigens are captured by dendritic cells and presented to
T lymphocytes in order to produce lymphoid factors, which act on primary tumor cells. Consequently, localized radiotherapy may have a abscopal effect in various areas [81]. PD-1 blockades amplify these abscopal effects, and radiotherapy increases the expression of PD-L1 in tumor cells [82] which suggests intervention compatibility. Therefore, early evidence around the radiotherapy with PD-1 blockade combination can also be considered promising.

Animal studies involving mice have shown that the median survival time of those receiving radiation plus a PD-1 blockade was 53 days which is twice that of the single-drug immunotherapy group [83]. In addition to these theoretical points, the PACIFIC study focused on patients with locally advanced lung cancer who had been treated with durvalumab for 1 year after concurrent radiotherapy and chemotherapy. The results suggest that survival can be substantially prolonged by 16.8 months when compared with placebo which was only 5.6 months [84]. Similarly, the results of a prospective phase I clinical trial involving patients with metastatic solid tumors suggest that stereotactic radiotherapy combined with a PD-1 inhibitor results in a 44\% ORR, with an mOS was 9.6 months and acceptable levels of toxicity [85].

Evidence from an increasing number of preclinical studies help rationalize and support combining radiotherapy with PD-1 blockades. However, there are only a few clinical studies focusing on this approach and most randomized clinical trials (RCTs) have been conducted with patients in the early stages of carcinoma development when responses may differ. Furthermore, knowledge around optimal radiotherapy dose, site location techniques, and interval between radiotherapy and PD-1 inhibitor treatments is lacking and must be explored in detail. In addition, insufficient is known about risk and harm associated with corresponding doses. Therefore while promising, we have a great deal to learn in devising appropriate PD-1 blockades combined with radiotherapy.

\section{PD-1 blockades combined with traditional chemotherapy}

A conventional strategy for enhancing the antitumor effect of immunotherapy is to combine with chemotherapy. Accumulating evidence indicates that chemotherapeutic drugs regulate the immune system while directly killing tumor cells by interfering with DNA synthesis and replication [86, 87]. Firstly, chemotherapy can induce immunogenic death of tumor cells because tumor-associated antigens are drained to the lymph nodes which hold the potential to increase the immune system's ability to identify tumors. Cytotoxic agents (i.e., taxanes) block tumor proliferation and affect innate immune cell function within the tumor microenvironment [88]. Second, the use of chemotherapeutic drugs can activate the interferon pathway of tumors, increasing $\mathrm{CD}^{+} \mathrm{T}$ cell infiltration while providing a suitable 
microenvironment for anti-PD-1/PD-L1 therapy [89]. In addition, chemotherapy might actually inhibit the immune escape mechanism of tumor cells by inhibiting MDSCs via selective depletion of Tregs [90, 91].

In a mouse model of lung adenocarcinoma, Pfirschke et al. found that autochthonous tumors which lacked $\mathrm{T}$ cell infiltration and resisted current treatment options could be sensitized to host antitumor $\mathrm{T}$ cell immunity when chemotherapy drugs are applied [92]. However, this initial evidence has only moderate support in human populations. The KEYNOTE-021 study involving patients with advanced non-squamous NSCLC found a 56.7\% ORR with pembrolizumab plus pemetrexed-Carboplatin (PC) compared with that of PC alone which was $30.2 \%$. Also, as a first-line treatment, pembrolizumab combined with PC has the potential to reduce the risk of disease progression by $44 \%$ with prolonged longevity compared with the PC control group, $24 \mathrm{mPFS}$ versus 9.3 month, respectively [93]. Due to fact that these participants were in the advanced stages of NSCLC, they had already received treatments which were not necessarily standardized. Logically, these treatments interact and are therefore likely to have added differential influences over the secondary treatment. Similar results have been found in patients with previously untreated metastatic non-squamous NSCLC without EGFR or ALK mutations. The results of the KEYNOTE-189 trial yielded a 69.2\%, 12-month overall survival (OS) for the pembrolizumab combination group. While in the placebo combination group, the 12-month OS was only $49.4 \%$ [94].

Again, many clinical studies are developing this evidence base around the efficacy of combined immunotherapies, though it remains necessary to monitor and report side effects. Furthermore, periodic administration of chemotherapeutic drugs may elicit a significant reduction in $\mathrm{T}$ lymphocytes which might weaken the immune effect of PD-1/PD-L1 blockades. It is therefore necessary to observe the $\mathrm{CD} 4^{+} / \mathrm{CD}^{+}$status and adjust dosages according to individual responses. The variety of chemotherapeutic drugs is subtly different, and there is significant heterogeneity among tumor types. Hence, investigating this combination as opposed to ICPIs or chemotherapy alone must be conducted according to tumor classification and characteristics. The mechanism of combination immunotherapies has been summarized in Fig. 2.

\section{Current applications of combination immunotherapy in gastrointestinal tumors}

Among the cluster of digestive tract tumors, histological differences are significant and are generally used to determine which approach to implement, especially for advanced tumors. For example, radiotherapy is efficacious in patients with esophageal cancer but not in patients with pancreatic cancer. Likewise, chemotherapy is the main stay for the treatment of patients with advanced gastric cancer but chemotherapeutic regimens are not generally administered for hepatocellular carcinoma. Therefore, combining superior interventions for digestive tract tumors with a single-drug immunotherapy may achieve enhanced immune expansion, despite the efficacy of PD-1/PD-L1 blockades varying substantially. Most studies are in the early phase clinical trials, although there are some which have progressed to phase III (Table 1). In this section, we systematically review officially published clinical studies for GI cancer sought through clinicaltrial.gov, PubMed, and in gray literature including conferences, such as ASCO and ESMO. Levels of efficacy will be critical discussed for several major digestive system tumors using relevant treatment indexes (i.e., OS, PFS, etc.).

\section{Esophageal carcinoma}

Moderate progress has been made in the diagnosis and treatment of esophageal cancer; however, the 5-year survival rate for patients with advanced esophageal cancer remains less than $15 \%$. A PD-1 blockade is mainly administered for patients with advanced esophageal cancer, including patients showing first-line drug resistant esophageal cancer, or localized progression and advanced metastasis. In the USA, pembrolizumab has been approved for the treatment of patients with chemotherapy-refractory PD-L1-positive gastroesophageal junction cancers on the basis of clinical activity observed in the KEYNOTE-059 trial. This study found that 95 patients, representing $42.4 \%$, experienced a reduction in measurable tumor size with a corresponding $11.6 \%$ ORR [95]. However, the KEYNOTE-180 study also found a $14 \%$ ORR for PD-1 blockages in esophageal squamous cell carcinoma patients compared with that of esophageal adenocarcinoma patients which was only $5 \%$. This finding was lower than had been expected given the findings in the KEYNOTE-28 study where the ORRs of squamous cell carcinoma and adenocarcinoma were $29 \%$ and $40 \%$, respectively [96, 97]. A subsequent phase III study, KEYNOTE-181 (NCT02564263), is currently looking to evaluate the activity of pembrolizumab versus a standard therapy in patients with metastatic esophageal carcinoma which progressed after receiving a first-line intervention. Preliminary outcomes suggest pembrolizumab is superior to chemotherapy for OS in PD-L1 with combined positive score $\geq 10$ patients. The reported 12 -month OS rate was $43 \%$ as opposed to $20 \%$, and drug-related AEs associated with pembrolizumab were fewer than in the group which received chemotherapy alone (64\% versus $86 \%)$.

ICPIs in esophageal cancer encourage optimism and combined with an immunotherapy may bring further benefit for those suffering esophageal cancer. Several clinical trials investigating PD-1 combined with radiotherapy have already been conducted with esophageal 


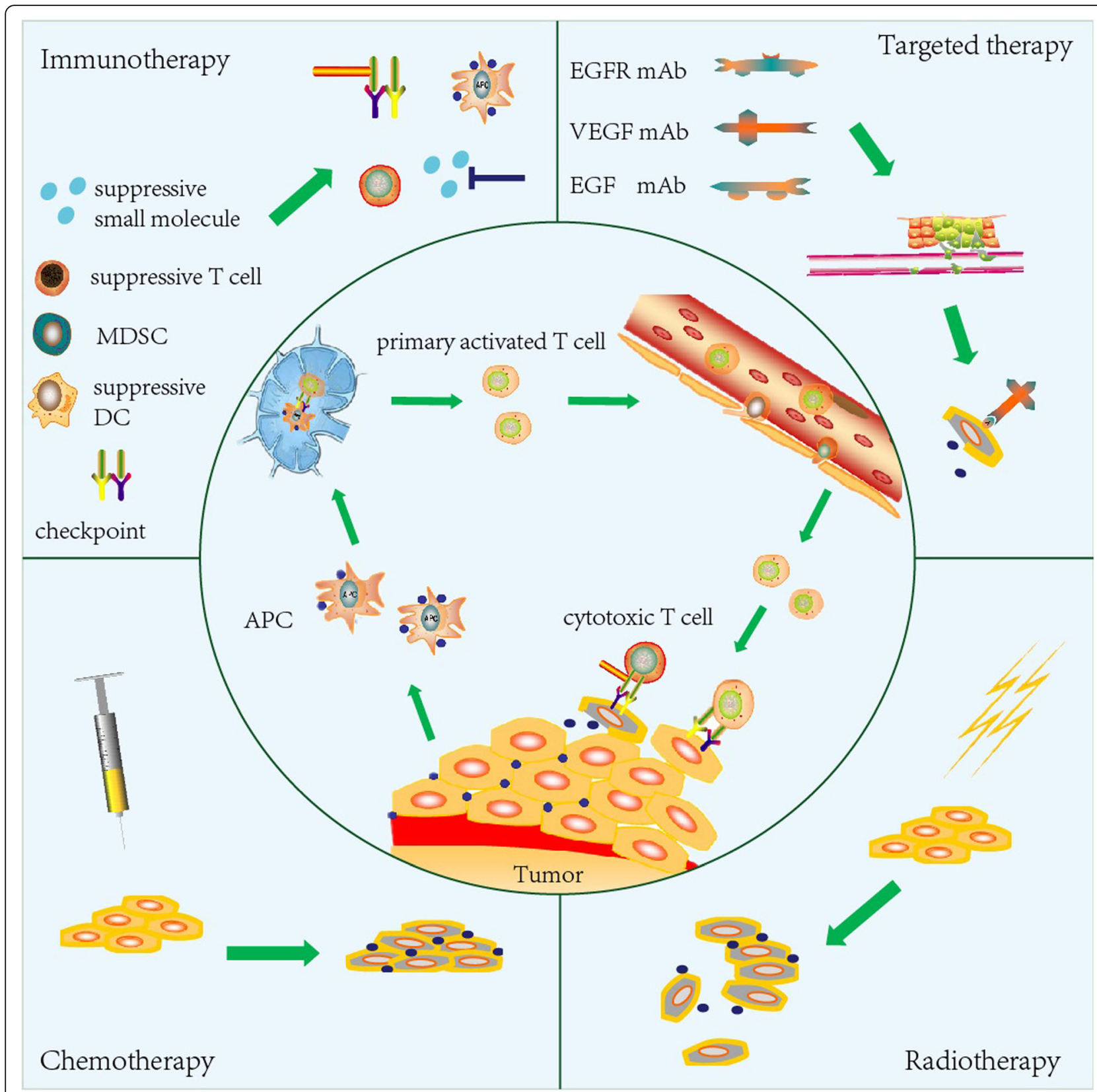

Fig. 2 Combination strategy in tumor immune circulation. As described in the cancer-immunity cycle, there are three main stages involving the presentation of tumor cell antigen by the APC cells, primary activation of T cells in the lymph node, and migration of cytotoxic T cells from the vessel to kill the tumor cells. Several other types of antitumor therapy, such as radiotherapy, chemotherapy, another immunotherapy, and targeted therapy, can participate in the cancer-immunity cycle by destroying the tumor matrix, increasing antigen exposure, removing the immunosuppressive factors, promoting the infiltration of T cells, etc.

cancer patients. The CheckMate-032 study focused on double immunotherapeutic interventions for esophageal cancer and found a $24 \%$ ORR for nivolumab administered at $1 \mathrm{mg} / \mathrm{kg}$ plus ipilimumab administered at $3 \mathrm{mg} / \mathrm{kg}$, with a corresponding PFS at 12 months of $17 \%$ (Table 2). This finding was twice that of the group which received nivolumab alone. However, the treatment-related grade 3 and 4 AEs in the combination group was $47 \%$, whereas with the single agent intervention resulted in only $17 \%$. The authors concluded that treatment with this ipilimumab combination significantly increased the incidence of side effects [98]. Finding an appropriate combination is clearly required, further necessitating the development of this evidence-base.

Based on current findings, a further phase III studies (NCT02872116) was designed to evaluate double 
Table 1 Ongoing phase 3 clinical trials of combined immunotherapy in gastrointestinal cancers

\begin{tabular}{|c|c|c|c|c|c|}
\hline Tumor type & $\begin{array}{l}\text { Phase/ } \\
\text { participants }\end{array}$ & $\begin{array}{l}\text { Immune } \\
\text { checkpoint } \\
\text { inhibitors } \\
\text { classification }\end{array}$ & Combination intervention & Status & $\begin{array}{l}\text { ClinicalTrials.gov } \\
\text { identifier }\end{array}$ \\
\hline $\begin{array}{l}\text { Unresectable, recurrent, locally } \\
\text { advanced or metastatic gastric or } \\
\text { gastroesophageal junction } \\
\text { adenocarcinoma }\end{array}$ & $3 / 371$ & $\begin{array}{l}\text { PD-L1 } \\
\text { inhibitors }\end{array}$ & Avelumab + BSC VS physician's choice + BSC & $\begin{array}{l}\text { Active, } \\
\text { not } \\
\text { recruiting }\end{array}$ & NCT02625623 \\
\hline Various advanced cancers & 3/939 & $\begin{array}{l}\text { PD-1 and CTLA- } \\
4 \text { inhibitors }\end{array}$ & $\begin{array}{l}\text { Nivolumab + ipilimumab or nivolumab + } \\
\text { fluorouracil + cisplatin VS fluorouracil + cisplatin }\end{array}$ & Recruiting & NCT03143153 \\
\hline Esophageal neoplasms & $3 / 700$ & PD-1 inhibitors & $\begin{array}{l}\text { Pembrolizumab + cisplatin and 5-fluorouracil (5- } \\
\text { FU) VS placebo + cisplatin and 5-FU }\end{array}$ & Recruiting & NCT03189719 \\
\hline $\begin{array}{l}\text { Esophageal carcinoma| } \\
\text { esophagogastric junction carcinoma }\end{array}$ & $3 / 720$ & PD-1 inhibitors & $\begin{array}{l}\text { Pembrolizumab (MK-3475) VS Investigator's } \\
\text { Choice Standard Therapy }\end{array}$ & $\begin{array}{l}\text { Active, } \\
\text { not } \\
\text { recruiting }\end{array}$ & NCT02564263 \\
\hline Gastric cancer & $3 / 700$ & PD-1 inhibitors & $\begin{array}{l}\text { Nivolumab + S-1 therapy or CapeOX therapy VS } \\
\text { placebo+ S-1 therapy or CapeOX therapy }\end{array}$ & Recruiting & NCT03006705 \\
\hline Gastric cancer & $2,3 / 680$ & PD-1 inhibitors & ONO-4538 + chemotherapy & $\begin{array}{l}\text { Active, } \\
\text { not } \\
\text { recruiting }\end{array}$ & NCT02746796 \\
\hline $\begin{array}{l}\text { Gastric cancer| gastroesophageal } \\
\text { junction cancer }\end{array}$ & $3 / 1649$ & $\begin{array}{l}\text { PD-1 and CTLA- } \\
4 \text { inhibitors }\end{array}$ & $\begin{array}{l}\text { Nivolumab + ipilimumab or nivolumab + } \\
\text { chemotherapy VS chemotherapy alone }\end{array}$ & Recruiting & NCT02872116 \\
\hline $\begin{array}{l}\text { Gastric cancer| Gastroesophageal } \\
\text { junction cancer }\end{array}$ & $3 / 860$ & PD-1 inhibitors & $\begin{array}{l}\text { Pembrolizumab (MK-3475) + chemotherapy VS } \\
\text { placebo + chemotherapy }\end{array}$ & Recruiting & NCT03221426 \\
\hline Stomach neoplasms & $3 / 780$ & PD-1 inhibitors & $\begin{array}{l}\text { Pembrolizumab (MK-3475) + chemotherapy VS } \\
\text { placebo + chemotherapy }\end{array}$ & $\begin{array}{l}\text { Not yet } \\
\text { recruiting }\end{array}$ & NCT03675737 \\
\hline $\begin{array}{l}\text { Gastric neoplasms| gastroesophageal } \\
\text { junction adenocarcinoma }\end{array}$ & $3 / 732$ & PD-1 inhibitors & $\begin{array}{l}\text { Pembrolizumab/Placebo + trastuzumab + } \\
\text { Chemotherapy }\end{array}$ & Recruiting & NCT03615326 \\
\hline Gastric adenocarcinoma & $3 / 764$ & PD-1 inhibitors & $\begin{array}{l}\text { Pembrolizumab as monotherapy, or } \\
\text { pembrolizumab + Cisplatin + 5-fluorouracil (5-FU) } \\
\text { or capecitabine; placebo + cisplatin + 5-FU or } \\
\text { capecitabine }\end{array}$ & $\begin{array}{l}\text { Active, } \\
\text { not } \\
\text { recruiting }\end{array}$ & NCT02494583 \\
\hline Biliary tract neoplasms & $3 / 390$ & PD-1 inhibitors & $\begin{array}{l}\text { KN035 + gemcitabine }+ \text { oxaliplatin VS } \\
\text { gemcitabine }+ \text { oxaliplatin }\end{array}$ & Recruiting & NCT03478488 \\
\hline Hepatocellular carcinoma & $3 / 330$ & PD-1 inhibitors & $\begin{array}{l}\text { Pembrolizumab (MK-3475) or placebo + Best } \\
\text { supportive care }\end{array}$ & Recruiting & NCT03062358 \\
\hline Hepatocellular Carcinoma & $3 / 480$ & PD-L1 inhibitors & Atezolizumab + bevacizumab VS sorafenib & Recruiting & NCT03434379 \\
\hline Hepatocellular carcinoma & $3 / 1200$ & $\begin{array}{l}\text { PD-L1 and } \\
\text { CTLA-4 } \\
\text { inhibitors }\end{array}$ & Durvalumab + tremelimumab & Recruiting & NCT03298451 \\
\hline Pancreatic cancer stage IV & $2 / 40$ & PD-1 inhibitors & $\begin{array}{l}\text { Nivolumab + cabiralizumab + gemcitabine VS } \\
\text { gemcitabine }\end{array}$ & $\begin{array}{l}\text { Not yet } \\
\text { recruiting }\end{array}$ & NCT03697564 \\
\hline Colorectal cancer & $3 / 363$ & PD-L1 inhibitors & $\begin{array}{l}\text { Cobimetinib + atezolizumab and atezolizumab } \\
\text { monotherapy VS regorafenib }\end{array}$ & $\begin{array}{l}\text { Active, } \\
\text { not } \\
\text { recruiting }\end{array}$ & NCT02788279 \\
\hline $\begin{array}{l}\text { Colorectal adenocarcinoma| } \\
\text { mismatch repair deficiency }\end{array}$ & $3 / 347$ & PD-L1 inhibitors & $\begin{array}{l}\text { Atezolizumab, bevacizumab, Mfolfox6 VS } \\
\text { bevacizumab, Mfolfox6 VS atezolizumab }\end{array}$ & Recruiting & NCT02997228 \\
\hline $\begin{array}{l}\text { Colon Adenocarcinomal DNA repair } \\
\text { disorder }\end{array}$ & $3 / 700$ & $\begin{array}{l}\text { PD-L1 and } \\
\text { CTLA-4 } \\
\text { inhibitors }\end{array}$ & $\begin{array}{l}\text { Combination chemotherapy with or without } \\
\text { atezolizumab }\end{array}$ & Recruiting & NCT02912559 \\
\hline Colorectal cancer & $3 / 180$ & PD-1 inhibitors & $\begin{array}{l}\text { Nivolumab with standard of care therapy VS } \\
\text { standard of care therapy for first-line treatment }\end{array}$ & Recruiting & NCT03414983 \\
\hline
\end{tabular}


immunotherapy as an early line therapy for esophagogastric cancers, and is presently under way. For the PD-1 and chemotherapy combination, the NCT03189719 trial is ongoing to evaluate the efficacy and safety of pembrolizumab plus cisplatin and 5-fluorouracil (5-FU) chemotherapy versus placebo plus cisplatin and 5-FU chemotherapy as a first-line treatment in participants with locally advanced or metastatic esophageal carcinoma. In fact, the majority of trials in this field are still in exploratory phases involving a variety of combinations. While results are pending, current knowledge provides some optimism and the results are eagerly anticipated.

\section{Gastric carcinoma}

The Cancer Genome Atlas (TCGA) divides gastric cancer into an Epstein-Barr virus (EBV) positive subtype, a microsatellite instability (MSI) subtype, a genomically stable (GS) subtype, and the chromosomal instability (CIN) subtype, according to histologically based integrative genomics [108]. Among the four types of gastric cancer, the high-frequency MSI (MSI-H) subtype appears to respond favorably [109]. The results of the ATTRACTION-02 phase III study focusing on heavily pretreated patients with advanced gastric or gastroesophageal junction cancer found OS rates in nivolumab compared with placebo were $27.3 \%$ and $11.6 \%$ at 12 months, and then $10.6 \%$ and $3.2 \%$ at 24 months, respectively. However, the nivolumab ORR was only in $11 \%$ of 268 patients which was considered a relatively low response rate [110].

Comparatively, the KEYNOTE-061 trial which focused on pembrolizumab with paclitaxel in patients with advanced gastric cancer whom had developed resistance after platinum and fluoropyrimidine treatment found that pembrolizumab did not significantly improve OS compared to paclitaxel, with an 9.1 month mOS versus 8.3 months [111]. Unsatisfactory immune monotherapies in gastric cancer make combined therapy especially enticing. Although, most of the combination strategies being investigated in gastric cancer are in the preclinical or early clinical research stage, few have entered the phase III stage [112]. For example, the CheckMate-649 is further assessing the difference in survival between nivolumab plus ipilimumab and chemotherapy although results are pending.

In the KEYNOTE-059 cohort 2 study, the ORR and DCR of 25 patients with advanced gastric or gastroesophageal adenocarcinoma were $60 \%$ and $80 \%$, and the median PFS and OS were 6.6 and 13.8 months, respectively. Subgroup analysis highlighted a 69\% ORR in PD-L1-positive patients and $38 \%$ in PD-L1-negative patients [99] (Table 2). This small sample study suggests that chemotherapy combined with anti-PD-1 has potential in gastric or gastroesophageal conjunctive adenocarcinoma, although confirmatory findings are required. In a related follow up, an investigation of the efficacy of chemotherapy combined with PD-1 blockades, KEYNOTE-062, is in progress to assess this combination as a first-line therapy for advanced gastric or gastroesophageal junction adenocarcinoma.

The preliminary results of a phase I/II study of ramucirumab plus nivolumab in patients with previously treated advanced gastric adenocarcinoma found a partial response was obtained in ten patients, representing a $22 \%$ of the study population with a DCR of $59 \%$ [100]. In addition, a phase I study (NCT02443324), which assessed the efficacy of pembrolizumab in combination with ramucirumab, found a 50\% DCR and PD-L1-positive patients appear to have significantly benefited [111]. Combination immunotherapies in esophageal and gastric cancer have achieved a preliminary advantage, and sequencing combination therapies is also moving forward.

\section{Hepatobiliary carcinoma}

Presently, targeted drugs, such as sorafenib, lenvatinib, and regorafenib, are the primary treatments for advanced hepatocellular carcinomas (HCC). Recent results have indicated the potential of PD-1/PD-L1 blockades for the treatment of advanced HCC. In the CheckMate-040 study, the overall ORR of the patients administered with nivolumab was $14-23 \%$. Subgroup analysis suggested that the DCR in patients without sorafenib was $54 \%$ with an OS of 28.6 months. In patients treated with sorafenib, the ORR was $55 \%$, suggesting that there may be only a fractional benefit, although this group had a prolonged 15.6 month OS [113]. In addition, liver toxicity of PD-1/PD-L1 blockades was lower than that of conventional drugs. As a result in 2017, nivolumab was approved by the FDA as a second-line treatment for HCC. Preliminary results from the KEYNOTE-224 study are similar to those of CheckMate-040, the ORR, and DCR in patients with advanced $\mathrm{HCC}$ whom had previously been treated with sorafenib was $17 \%$ and $61 \%$, respectively [114]. In view of the aforementioned findings, the phase III CheckMate- 459 trial which will compare nivolumab with sorafenib as first-line treatments for advanced HCC with overall survival as the primary endpoint is much needed [115].

PD-1 inhibitor monotherapies appear to be well tolerated with relatively consistent efficacy in liver cancer patients. For example, the retrospective study of CheckMate-040 trial found a 50\% ORR in 14 patients whom had received nivolumab combined with localregional treatment with three CRs (11\%) and five PRs (18\%) [116]. To further increase the antitumor response, a preliminary study of lenvatinib plus pembrolizumab in patients with unresectable HCC resulted in encouraging antitumor activity and tolerance with $46 \%$ ORR (Table 3 ). 


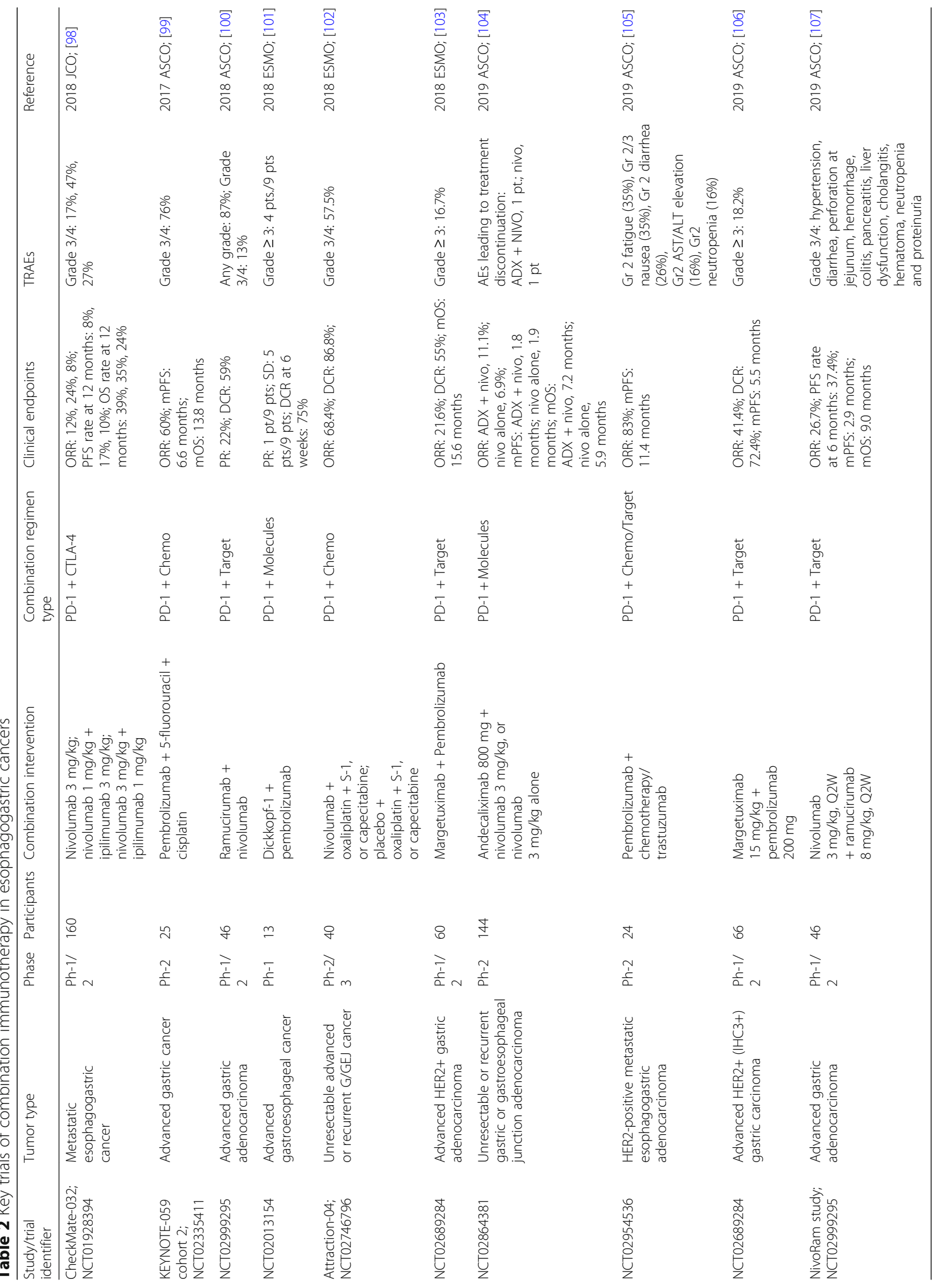


The most common AEs were decreased appetite and hypertension without new safety signals [117].

The FDA recommends atezolizumab combined with bevacizumab as a first-line therapeutic regimen for patients with advanced HCC based on a phase 1b study (NCT02715531). The findings of this study highlight a $34 \%$ ORR associated with atezolizumab combined with bevacizumab among 68 patients assessed [118], although this was a relatively small study. The recent phase III IMbrave150 trial is based upon these findings and will evaluate the efficacy and safety of this combination compared to sorafenib in participants with locally advanced or metastatic HCC who have received no prior systemic treatment [127]. Combining PD-1 blockade and CTLA-4 blockade for advanced HCC may also prove beneficial and early data from NCT02519348 suggests relative safety with an 18\% ORR [113] and the upgraded study is currently recruiting. In addition, several clinical trials of PD-1/PD-L1 blockades combined with other types of antitumor therapy are also under way.

Related basic medical research by Nakamura et al. divided biliary tract cancers (BTC) into four molecular subgroups based upon prognostic gene profiles and found that classification correlates with patient prognosis. Among subtypes with the worst prognosis, the expression of immune checkpoint-related molecules, including PD-L1, was upregulated more than in any other subgroups, which again suggests immune checkpoint inhibitors may yield a favorable response [128]. In addition, emerging data suggests MMR or MSI-H mutation tumors have a much higher response rate to PD-1/ L1 inhibitors, and in cholangiocarcinoma, MSI-H accounting for $5 \%$ of gallbladder cancers (GBC), 5-13\% of extrahepatic cholangiocarcinoma (ECC), and $10 \%$ of intrahepatic cholangiocarcinoma (ICC) [109]. Phage 1b KEYNOTE-028 trail assessed the safety and activity of pembrolizumab monotherapy among advanced solid tumors with PD-L1 expression $\geq 1 \%$, and the cholangiocarcinoma cohort suggested that of 24 patients who met the evaluation criteria ORR was 17\% [129].

Sequencing exons and transcriptomes has revealed heterogeneous molecular changes among cholangiocarcinoma, and the selection of an immunotherapy combined with a targeted therapy may provide answers where other avenues may not. One small sample study found after treatment with PD-1 blockades combined with lenvatinib, $3: 14$ patients had a $21.4 \%$ ORR and a 93\% DCR. Interestingly, this study using 450 -gene next generation sequencing (NGS) panel in seven patients to detect all classes of genetic status discovered that having a high TMB might be used to indicate preferential treatment [121] (Table 3). The standard first-line chemotherapy for advanced BTC is gemcitabine plus cisplatin; however, there is no standardized second-line intervention. This is because evidence is lacking to guide specialists. PD-1/L1 blockades combined with a standard chemotherapy is frequently administered as a secondline therapy, although there appears to be an element of trial and error adjustment. Currently, several clinical trials are under way, including one investigating a guadecitabine and durvalumab combination (NCT03257761) and another pembrolizumab and FOLFOX (NCT02268825) (Table 3). The findings of these studies may provide support for clinicians seeking the most effective option where first-line treatments have failed.

Another interesting research avenue which has emerged is around the impact of standards of care (SoC). Currently under way, a phase III clinical study is exploring this in more detail, focusing on the efficacy of PD-1 blockade combined with SoC compared with SoC alone for the treatment of previously untreated locally advanced or metastatic BTC. The primary hypothesis of the study is that participants will have a longer OS when treated with combined therapy than when treated with SoC alone, although this study may also provide insight into the interactions between $\mathrm{SoC}$ and PD-1 blockades which is also needed.

\section{Pancreatic carcinoma}

Previously presented evidence suggests that immunotherapy combined with PD-1/PD-L1 blockades may deliver favorable outcomes with durable responses for various types of cancer; however, pancreatic carcinomas remain refractory. Except for MSI-positive pancreatic cancers which accounts for approximately $1.2 \%$, the efficacy of PD-1/PD-L1 blockades alone are unsatisfactory for most pancreatic cancers. Unfortunately, more than $10 \%$ of patients develop grade 3 and 4 AEs, which is likely to be at least partly be due to the unique microenvironments (TME) in the pancreas [130]. Pancreatic tumor tissues are characterized by excessive cancerassociated fibroblasts (CAFs), dense connective tissue, low vascular density, and insensitivity to ischemia and hypoxia. In addition, immunosuppressive immune cells, such as M2 macrophages, are found in tumor tissues which inhibit immune killer cells from effectively entering through the tumor matrix [131]. Potentially, combined immunotherapies could provide a solution to these problems by bolstering the immune response to pancreatic tumor development.

Presently, gemcitabine, albumin paclitaxel, and a monoclonal CD40 antibody combined with nivolumab are frequently used as pancreatic cancer interventions. These interventions act by destroying tumor matrices and by exposing more antigens, which promote lymphocyte infiltration. Cabiralizumab (FPA008) is an antiCSF-1R antibody which can cause the depletion of tumor-associated macrophages (TAMs) which may 


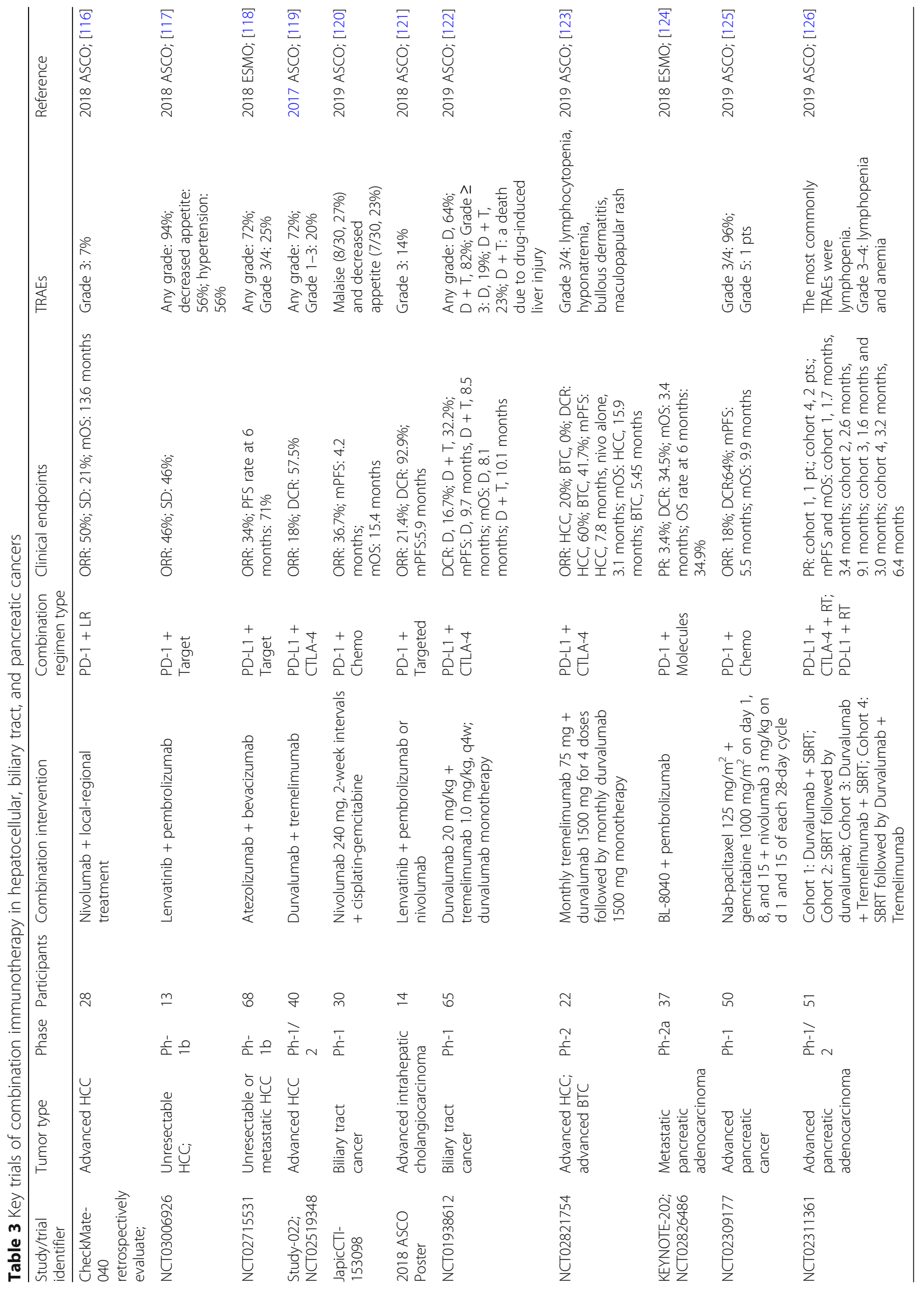


provide additional benefit. As such, one recent study (NCT02526017) was designed specifically to evaluate the safety, tolerability, as well as the clinical benefit of cabiralizumab in combination with nivolumab in patients with selected advanced cancers, including pancreatic cancer. The study revealed lasting clinical benefit for five patients with advanced pancreatic cancer who were insensitive to a previously administered single-drug immunotherapy, including three patients with microsatellite stability (MSS). However, the sample size of the study was small $(n=33)$, therefore these results ought be verified under stricter conditions, including a larger sample size based on a pre-trial calculation using best available evidence, and with an appropriate control group. Importantly, it is necessary to conduct this research focusing on those suffering pancreatic cancer specifically because of the refractory nature of this condition but also to explore therapeutic effects across stages.

A phase II clinical trial (NCT03336216) currently under way is focusing on the efficacy of cabiralizumab and nivolumab combined with or without chemotherapy specifically for the treatment of advanced pancreatic cancer. Chemotherapy in this particular trial includes paclitaxel, gemcitabine, irinotecan, or FOLFIRINOX. The researchers have proposed to recruit 160 patients which is substantially larger than previously mentioned NCT02526017 study, and to use PFS as the primary clinical endpoint. The potential benefit of PD-1/PD-L1 blockades combined with other therapeutic approaches has resulted in a number of trials focusing on resectable pancreatic cancer, broad line resectable pancreatic cancer, and advanced pancreatic cancer. Most of the trials being designed are again preclinical studies or early phase clinical research but hopefully findings from the aforementioned studies will develop this evidence base and drive higher level clinical research.

\section{Colorectal carcinoma}

The KEYNOTE-028 trial which involved a cohort of people with existing colon and rectum carcinomas found only a $4 \%$ ORR for pembrolizumab monotherapy after screening out patients with PD-L1 $>1 \%(n=1)$, and there was no significant improvement when compared with that of unscreened patients [132]. DMMR/MSI-Htype mCRC accounts for $4 \%$ of mCRC overall, although this is insensitive to traditional chemotherapy and generally has a poor prognosis. However, many neoantigens increase dMMR patients' sensitivity to PD-1/PD-L1 blockade therapy. Therefore, nivolumab has been approved for patients with metastatic DNA mismatch repair-deficient colorectal cancer based on the Checkmate 142 study suggesting 23 of 74 patients achieved objective response and $68.9 \%$ of patients had disease control for $\geq 12$ weeks [133].

Nevertheless, the colorectal cancer group of phase II clinical trials evaluating the clinical activity of pembrolizumab in patients with progressive metastatic carcinoma has shown that the ORR and DCR of patients with mismatch repair-deficient (dMMR) within 20 weeks were 40 and $90 \%$, respectively. For the mismatch repair-proficient (pMMR) group, these values were 0 and $11 \%$, respectively which suggests that mismatch repair status may be used as efficient indicators of PD-1 antibodies, although further research is needed for clarification [109]. One phase 3 clinical trial (NCT02563002) has been designed to investigate these issues and will compare PFS and OS between dMMR/MSI-H patients administered single-drug PD-1 inhibitor therapy and dMMR/ MSI-H patients administered standard chemotherapy.

Concerning double immunotherapy in dMMR/MSI-H $\mathrm{mCRC}$, results for the nivolumab plus ipilimumab cohort of CheckMate-142 study found at the median follow-up (13.4 months) a 55\% ORR with corresponding PFS and OS rates at 12 months of $76 \%$ and $87 \%$, respectively [134] (Table 4). Therefore, indirect comparisons suggest that combination therapies provide improved efficacy relative to anti-PD-1 monotherapy (ORR 31\%) and has a favorable benefit-risk profile. Importantly, the study also suggests that there is no relationship between efficacy and the expression of PD-L1 in MSI-H patients.

As mentioned previously, PD-1 inhibitor monotherapy has little effect in patients with microsatellite stable colorectal cancer. Indeed, many factors may influence the efficacy of PD-1/PD-L1 blockade in patients with colorectal cancer, including gene mutations, the immune microenvironment, and a patient's genetic inheritance. In unscreened patients with advanced colorectal cancer, a small sample study at the 24-week follow-up found 53\% ORR for PD-1 blockade combined with chemotherapy. Although, it remains unclear how effective chemotherapy alone will be for this group of patients due to the lack of rigorous experimental design, and the proportion of people (36.7\%) suffering associated severe side effects associated [135].

MEK inhibition upregulates tumor major histocompatibility complex-I expression, promoting intra-tumoral $\mathrm{T}$ cell accumulation while improving anti-PD-L1 responses [140]. For patients with MSS colorectal cancer, recent studies have found that cobimetinib (MEK1/2 inhibitor) combined with PD-L1 blockades results in a DCR of $31 \%$, and $43 \%$ of patients survive for more than 12 months [136]. As a result, a phase III clinical trial (NCT02788279) was designed to evaluate atezolizumab in combination with cobimetinib versus atezolizumab or regorafenib monotherapies and the findings are eagerly anticipated. 


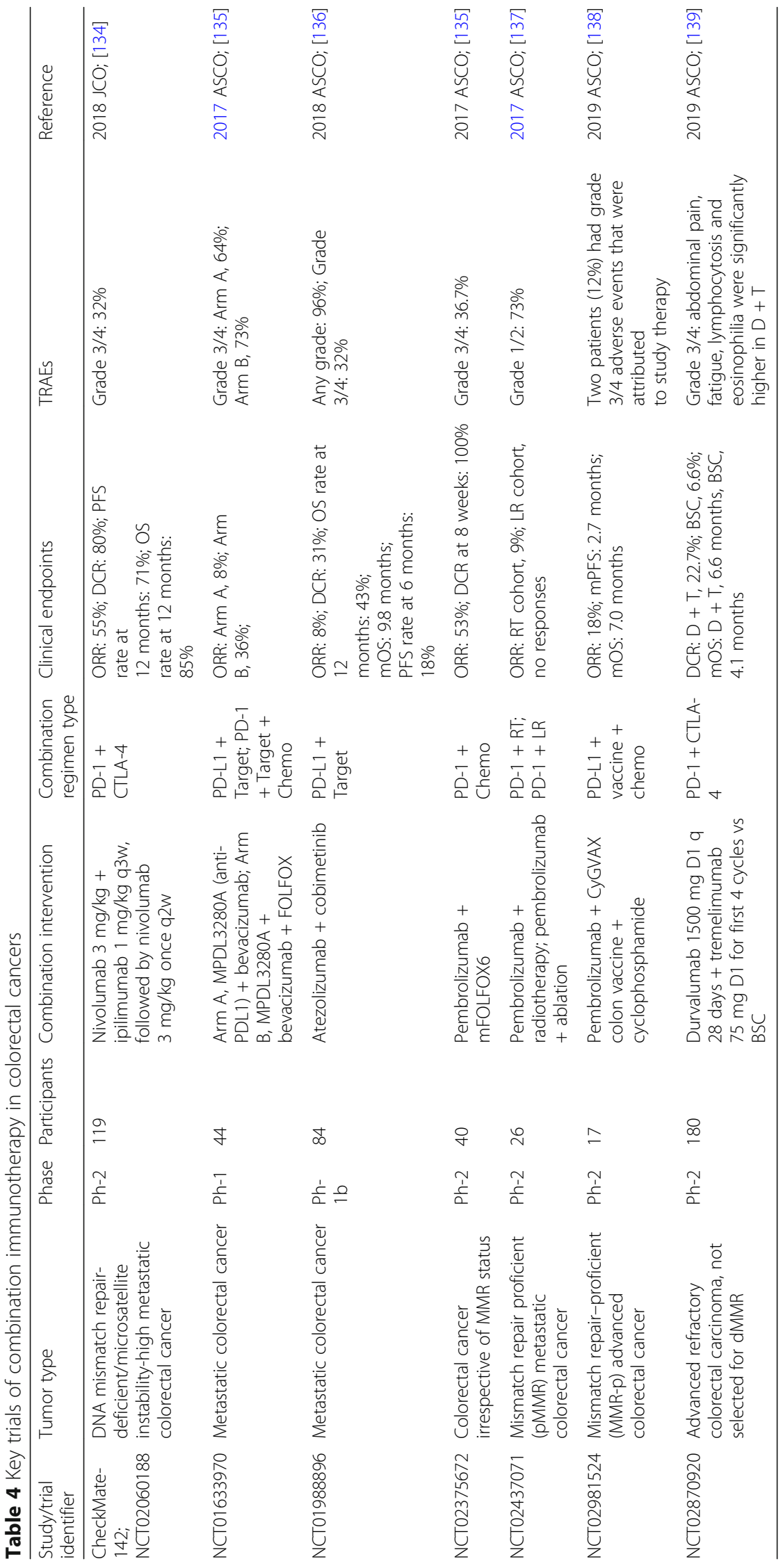


An increasing number of clinical trials are currently under development and ongoing which provides some optimism. However, these combinations face a number of problems, such as the need for more comprehensive gene sequencing and the difficulty of accurately and rigorously classifying colorectal cancer patients to predict treatment efficacy. In addition, the use of the same treatment regimen for different patients may not improve prognoses due to significant differences among individual patients which suggest the need for personalized cancer care. However, in order for this to become a reality, studies need to be scaled up and studies ought to be designed to incorporate the subtle differences between participants, which one could argue is not the current state of play.

\section{Conclusions and perspectives}

The advantages of combined immunotherapy based on PD-1/PD-L1 blockades for various tumors appear to be the logical next step. Although, there are a great number of unknowns, including dose/response, safety, tolerability, durability, and indeed efficacy. How these new treatment options will be placed within the existing treatment framework is a concern. Researchers are endeavoring to answer these questions through rigorous clinical trials focusing on specific types of tumors and within specific populations at various stages of these diseases. Studies have found an increase in the proportion of immune-related adverse events after receiving combination therapy compared to monotherapies. Although, these generally include diarrhea, fatigue, and hypothyroidism, which are within a tolerable range and manageable [17].

The increasing the number of combination studies has highlighted beneficial antitumor effects in early clinical stages. However, results from several clinical trials found no enhanced benefit for the patients with advanced cancers. Moreover, administering combination immunotherapies has been found to increase treatment toxicity. In patients who received radiotherapy prior to treatment with PD-1 blockades, research has revealed that immune inflammation frequently and naturally recurs at the original site of irradiation. Therefore, as many of the current combined immunotherapeutic methods remain experimental, developing this evidence base is absolutely necessary.

Understanding the underlying mechanisms of each therapeutic combination as well as the subtleties of individual responses is required to avoid combination schemes which do harm. Ironically, combination immunotherapeutic models pose similar questions to traditional treatment: What is the ideal patient population for which combination? Is the required combination therapy sequential or concurrent? What timing and adjustment criteria can be used for continuous and combined interventions? What is the related safety and toxicity of each combination? All of these questions require a sophisticated evidence-base developed through mature theoretical foundations and basic medical research. Once small sample studies have been conducted, larger studies ought to be commenced as is currently occurring. However, at present, it would appear as though we are trying to improve outcomes by combining a possible best available treatment with a potential catalyst or less subtly, simply seeking compatible combinations. We must not overlook the fact that this is essentially combining an average of averages with yet another. More specific research is required with more comprehensive data collection if we are to treat individuals with more precision and sensitivity as is required for gastrointestinal malignancies. Further research should focus on markers as these may provide measurable trajectories to accurately predict the benefit of combination therapies.

\section{Abbreviations}

5-FU: 5-Fluorouracil; AEs: Adverse events; APCs: Antigen-presenting cells; ASCO: American Society of Clinical Oncology; B2M: Beta-2-microglobulin; B7H1: B7 homolog 1; BTC: Biliary tract cancer; CAFs: Cancer-associated fibroblasts; CCL5: Cell chemokine ligand 5; CIN: Chromosomal instability; CRC: Colorectal cancer; CTLA4: Cytotoxic T lymphocyte antigen-4; CXCL10: CX-C motif chemokine 10; CXCR2: C-X-C Motif chemokine Receptor 2; DCR: Disease control rate; dMMR: Mismatch repair deficient; DOR: Duration of response; EBV: Epstein-Barr virus; EC: Esophagus cancer; ECC: Extrahepatic cholangiocarcinoma; ECCO: European Cancer Congress; EGFR: Epidermal growth factor receptor; FDA: Food and Drug Administration;

GBC: Gallbladder cancer; GC: Gastric cancer; GEC: Gastroesophageal junction cancer; GI: Gastrointestinal; GITR: Glucocorticoid-induced TNFR family related gene; GS: Genomically stable; HCC: Hepatocellular carcinoma; HDAC: Histone deacetylase; HLA: Human leukocyte antigen; ICC: Intrahepatic

cholangiocarcinoma; ICPIs: Immune checkpoint inhibitors; IDO: Indoleamine 2,3-dioxygenase; INV: Investigator; irAEs: Immune-related adverse events; JAK: Janus kinases; LAG-3: Lymphocyte-activation gene 3; MANAs: Mutationassociated neoantigens; McAbs: Monoclonal antibodies; MDM2/4: Murine double minute 2/4; MDSCs: Myeloid-derived suppressor cells; MEK: MAP kinse-ERK kinase; MHC: Major histocompatibility complex; mOS: Median overall survival; MSI-H: Microsatellite instability-high; MSS: Microsatellite stability; NSCLC: Non-small cell lung cancer; ORR: Objective response rate; OS: Overall survival; P13K: Phosphoinositide 3-kinase; PC: Pancreatic cancer; PD: Progressive disease; PD-1: Programmed cell death-1; PD-L1: Programmed cell death ligand-1; PD-L2: Programmed cell death ligand-2; PFS: Progressionfree survival; pMMR: mismatch repair proficient; PR: Partial response; RCTs: Randomized clinical trials; SCCA: Squamous cell carcinoma of the anal canal; SCCs: Squamous cell carcinomas; SD: Stable disease; TAMs: Tumorassociated macrophages; TCGA: The Cancer Genome Atlas; TCR: T cell receptor; TILs: Tumor infiltrating lymphocytes; TIM-3: T cell immunoglobulin mucin-3; TMB: Tumor mutational burden; TME: Tumor microenvironment; TNFRSF4: Tumor necrosis factor receptor super family member 4; TRAEs: Treatment-related adverse events; Tregs: T regulatory cells; TRTEAEs: Treatment-related treatment-emergent adverse events; VEGF: Vascular endothelial growth factor

\section{Acknowledgements}

Not applicable.

\section{Funding}

This work was supported by International Science and Technology Cooperation Projects (2016YFE0107100 and 2015DFA30650), CAMS Innovation Fund for Medical Science (CIFMS) (2017-I2M-4-003), Capital Special Research Project for Health Development (2014-2-4012), Beijing Natural Science Foundation (L172055), and National Ten-thousand Talent Program and Beijing Science and Technology Cooperation Special Award Subsidy Project. CAMS Initiative for Innovative Medicine (CAMS-2018-12M-3-001). 


\section{Availability of data and materials}

All data generated or analyzed during this study are included in this published article and its supplementary information files.

\section{Authors' contributions}

$D W, J L$ and $X Y$ searched the literature and wrote the manuscript. $J L, Y B$ and $\mathrm{XY}$ helped to collect literature and participated in discussions. YM, XS and $\mathrm{HZ}$ designed the study. SS and HZ examined and verified the study. All authors read and approved the final manuscript.

\section{Ethics approval and consent to participate}

Not applicable.

\section{Consent for publication}

Not applicable.

\section{Competing interests}

The authors declare that they have no competing interests.

\section{Publisher's Note}

Springer Nature remains neutral with regard to jurisdictional claims in published maps and institutional affiliations.

\section{Author details}

${ }^{1}$ Department of Liver Surgery, Peking Union Medical College Hospital, Chinese Academy of Medical Sciences and Peking Union Medical College, Beijing, China. ${ }^{2}$ Department of Humanities, Peking Union Medical College, Chinese Academy of Medical Sciences and Peking Union Medical College, Beijing, China.

Received: 18 February 2019 Accepted: 2 April 2019

Published online: 24 April 2019

\section{References}

1. Bray F, Ferlay J, Soerjomataram I, Siegel RL, Torre LA, Jemal A. Global cancer statistics 2018: GLOBOCAN estimates of incidence and mortality worldwide for 36 cancers in 185 countries. CA Cancer J Clin. 2018;68(6):394-424.

2. Papaioannou NE, Beniata OV, Vitsos P, Tsitsilonis O, Samara P. Harnessing the immune system to improve cancer therapy. Ann Transl Med. 2016;4(14):261.

3. Alsaab HO, Sau S, Alzhrani R, Tatiparti K, Bhise K, Kashaw SK, lyer AK. PD-1 and PD-L1 checkpoint signaling inhibition for cancer immunotherapy: mechanism, combinations, and clinical outcome. Front Pharmacol. 2017:8:561.

4. Quail DF, Joyce JA. Microenvironmental regulation of tumor progression and metastasis. Nat Med. 2013;19(11):1423-37.

5. Bindea G, Mlecnik B, Angell HK, Galon J. The immune landscape of human tumors: implications for cancer immunotherapy. Oncoimmunology. 2014;3(1):e27456.

6. Hazama S, Tamada K, Yamaguchi Y, Kawakami Y, Nagano H. Current status of immunotherapy against gastrointestinal cancers and its biomarkers: perspective for precision immunotherapy. Ann Gastroenterol Surg. 2018;2(4):289-303.

7. Long J, Lin J, Wang A, Wu L, Zheng Y, Yang X, Wan X, Xu H, Chen S, Zhao H. PD-1/PD-L blockade in gastrointestinal cancers: lessons learned and the road toward precision immunotherapy. J Hematol Oncol. 2017;10(1):146.

8. Iwai Y, Ishida M, Tanaka Y, Okazaki T, Honjo T, Minato N. Involvement of PD-L1 on tumor cells in the escape from host immune system and tumor immunotherapy by PD-L1 blockade. Proc Natl Acad Sci USA. 2002;99(19):12293-7.

9. Sanmamed MF, Chen L. A paradigm shift in cancer immunotherapy: from enhancement to normalization. Cell. 2018;175(2):313-26.

10. Strome SE, Dong H, Tamura H, Voss SG, Flies DB, Tamada K, Salomao D, Cheville J, Hirano F, Lin W, et al. B7-H1 blockade augments adoptive T-cell immunotherapy for squamous cell carcinoma. Cancer Res. 2003;63(19):6501-5.

11. Topalian SL, Hodi FS, Brahmer JR, Gettinger SN, Smith DC, Mcdermott DF, Powderly JD, Carvajal RD, Sosman JA, Atkins MB. Safety, activity, and immune correlates of anti-PD-1 antibody in cancer; 2012.

12. Gettinger $S$, Horn $L$, Jackman D, Spigel D, Antonia S, Hellmann M, Powderly J, Heist R, Sequist LV, Smith DC, et al. Five-year follow-up of nivolumab in previously treated advanced non-small-cell lung cancer: results from the CA209-003 study. J Clin Oncol. 2018;36(17):1675-84.
13. Xu-Monette ZY, Zhang M, Li J, Young KH. PD-1/PD-L1 blockade: have we found the key to unleash the antitumor immune response? Front Immunol. 2017:8:1597

14. Ma W, Gilligan BM, Yuan J, Li T. Current status and perspectives in translational biomarker research for PD-1/PD-L1 immune checkpoint blockade therapy. J Hematol Oncol. 2016;9(1):47.

15. Wang Q, Wu X. Primary and acquired resistance to PD-1/PD-L1 blockade in cancer treatment. Int Immunopharmacol. 2017;46:210-9.

16. Flynn M J, Larkin J M G. Novel combination strategies for enhancing efficacy of immune checkpoint inhibitors in the treatment of metastatic solid malignancies[J]. Expert Opin Pharmacother. 2017;18(14):1477-90.

17. Postow MA, Sidlow R, Hellmann MD. Immune-related adverse events associated with immune checkpoint blockade. N Engl J Med. 2018; 378(2):158-68.

18. Topalian SL, Hodi FS, Brahmer JR, Gettinger SN, Smith DC, McDermott DF, Powderly JD, Carvajal RD, Sosman JA, Atkins MB. Safety, activity, and immune correlates of antiPD-1 antibody in cancer. N Engl J Med. 2012;366(26):2443-54.

19. Sandigursky S, Mor A. Immune-related adverse events in cancer patients treated with immune checkpoint inhibitors. Curr Rheumatol Rep. 2018;20(10):65.

20. Spain L, Diem S, Larkin J. Management of toxicities of immune checkpoint inhibitors. Cancer Treat Rev. 2016;44:51-60.

21. Haanen J, Carbonnel F, Robert C, Kerr KM, Peters S, Larkin J, Jordan K. Management of toxicities from immunotherapy: ESMO clinical practice guidelines for diagnosis, treatment and follow-up. Ann Oncol. 2017; 28(suppl_4):iv119.

22. Chen JH, Pezhouh MK, Lauwers GY, Masia R. Histopathologic features of colitis due to immunotherapy with anti-PD-1 antibodies. Am J Surg Pathol. 2017;41(5):643-54

23. Dougan M. Checkpoint blockade toxicity and immune homeostasis in the gastrointestinal tract[J]. Front Immunol. 2017;8:1547. https://doi.org/10.3389/ fimmu.2017.01547.

24. Hofmann L, Forschner A, Loquai C, Goldinger SM, Zimmer L, Ugurel S, Schmidgen MI, Gutzmer R, Utikal JS, Göppner D. Cutaneous, gastrointestinal, hepatic, endocrine, and renal side-effects of anti-PD-1 therapy. Eur J Cancer. 2016;60:190-209.

25. Moslehi JJ, Salem J-E, Sosman JA, Lebrun-Vignes B, Johnson DB. Increased reporting of fatal immune checkpoint inhibitor-associated myocarditis. Lancet. 2018;391(10124):933.

26. Sato K, Akamatsu H, Murakami E, Sasaki S, Kanai K, Hayata A, Tokudome N, Akamatsu K, Koh Y, Ueda H, et al. Correlation between immune-related adverse events and efficacy in non-small cell lung cancer treated with nivolumab. Lung Cancer (Amsterdam, Neth.). 2018;115:71-4.

27. Milano G. Resistance to immunotherapy: clouds in a bright sky. Invest New Drugs. 2017;35(5):649-54.

28. Beaver JA, Hazarika M, Mulkey F, et al. Patients with melanoma treated with an anti-PD-1 antibody beyond RECIST progression: a US Food and Drug Administration pooled analysis[J]. Lancet Oncol. 2018;19(2):229-39.

29. Zaretsky JM, Garcia-Diaz A, Shin DS, Escuin-Ordinas H, Hugo W, HuLieskovan S, Torrejon DY, Abril-Rodriguez G, Sandoval S, Barthly L, et al. Mutations associated with acquired resistance to PD-1 blockade in melanoma. N Engl J Med. 2016;375(9):819-29.

30. Koyama S, Akbay EA, Li YY, Herter-Sprie GS, Buczkowski KA, Richards WG, Gandhi L, Redig AJ, Rodig SJ, Asahina H. Adaptive resistance to therapeutic PD-1 blockade is associated with upregulation of alternative immune checkpoints. Nat Commun. 2016;7:10501.

31. Champiat S, Dercle L, Ammari S, Massard C, Hollebecque A, Postel-Vinay S, Chaput N, Eggermont A, Marabelle A, Soria JC. Hyperprogressive disease is a new pattern of progression in cancer patients treated by anti-PD-1/PD-L1. Clin Cancer Res. 2017;23(8):1920-8.

32. Saada-Bouzid E, Defaucheux C, Karabajakian A, Coloma VP, Servois V, Paoletti X, Even C, Fayette J, Guigay J, Loirat D, et al. Hyperprogression during anti-PD-1/PD-L1 therapy in patients with recurrent and/or metastatic head and neck squamous cell carcinoma. Ann Oncol. 2017;28(7):1605-11.

33. Shinozaki T, Iwami E, Ikemura S, Matsuzaki T, Nakajima T, Hashimoto K, Terashima T. A case of pulmonary adenocarcinoma showing rapid progression of peritoneal dissemination after immune checkpoint inhibitor therapy. BMC Cancer. 2018;18(1):620.

34. Ferrara R, Caramella C, Texier M, et al. 1306PDHyperprogressive disease (HPD) is frequent in non-small cell lung cancer (NSCLC) patients (pts) treated with anti PD1/PD-L1 monoclonal antibodies (IO)[J]. Ann Oncol. 2017;28:mdx380.009. https://doi.org/10.1093/annonc/mdx380.009. 
35. Kato S, Goodman A, Walavalkar V, et al. Hyperprogressors after immunotherapy: analysis of genomic alterations associated with accelerated growth rate[J]. Clin Cancer Res. 2017;23(15): 4242-50.

36. Topalian SL, Taube JM, Anders RA, Pardoll DM. Mechanism-driven biomarkers to guide immune checkpoint blockade in cancer therapy. Nat Rev Cancer. 2016;16(5):275.

37. Herbst RS, Soria J-C, Kowanetz M, Fine GD, Hamid O, Gordon MS, Sosman JA, McDermott DF, Powderly JD, Gettinger SN. Predictive correlates of response to the anti-PD-L1 antibody MPDL3280A in cancer patients. Nature. 2014;515(7528):563.

38. Tremblay-Lemay R, Rastgoo N, Chang H. Modulating PD-L1 expression in multiple myeloma: an alternative strategy to target the PD-1/PD-L1 pathway. J Hematol Oncol. 2018;11(1):46.

39. Tray N, Weber JS, Adams S. Predictive biomarkers for checkpoint immunotherapy: current status and challenges for clinical application. Cancer Immunol Res. 2018;6(10):1122-8

40. Yarchoan M, Hopkins A, Jaffee EM. Tumor mutational burden and response rate to PD-1 inhibition. N Engl J Med. 2017;377(25):2500.

41. Berntsson J, Larsson A, Nodin B, Eberhard J, Jirstrom K. Prognostic impact of PD-L1 and PD-1 expression by primary tumor location in colorectal cancer. J Clin Oncol. 2018;36(4_suppl):628.

42. Dunn GP, Bruce AT, Ikeda H, Old L, Schreiber RD. Cancer immunoediting: from immunosurveillance to tumor escape. Nat Immunol. 2002:3(11):991-8.

43. Chen D, Mellman I. Oncology meets immunology: the cancer-immunity cycle. Immunity. 2013;39(1):1-10.

44. Coulie PG, Van BDE, Van PDB, Boon T. Tumour antigens recognized by T lymphocytes: at the core of cancer immunotherapy. Nat Rev Cancer. 2014;14(2):135-46.

45. Facciabene A, Motz GT, Coukos G. T-regulatory cells: key players in tumor immune escape and angiogenesis. Cancer Res. 2012;72(9):2162-71.

46. Ok CY, Young KH. Checkpoint inhibitors in hematological malignancies. J Hematol Oncol. 2017;10(1):103

47. Binnewies M, Roberts EW, Kersten $K$, Chan V, Fearon DF, Merad M, Coussens LM, Gabrilovich DI, Ostrand-Rosenberg S, Hedrick CC, et al. Understanding the tumor immune microenvironment (TIME) for effective therapy. Nat Med. 2018;24(5):541-50.

48. Galon J, Bruni D. Approaches to treat immune hot, altered and cold tumours with combination immunotherapies. Nat Rev Drug Discov. 2019;18: 197-218.

49. Jbag H. Converting cold into hot tumors by combining immunotherapies. Cell. 2017;170(6):1055-6. https://doi.org/10.1038/s41573-018-0007-y.

50. Pitt JM, Vétizou M, Daillère $R$, Roberti MP, Yamazaki T, Routy $B$, Lepage $P$ Boneca IG, Chamaillard M, Kroemer G. Resistance mechanisms to immunecheckpoint blockade in cancer: tumor-intrinsic and -extrinsic factors. Immunity. 2016;44(6):1255-69.

51. Chen G, Emens LA. Chemoimmunotherapy: reengineering tumor immunity. Cancer Immunol Immunother. 2013:62(2):203-16.

52. Zhang B, Wu Q, Zhou YL, Guo X, Ge J, Fu J. Immune-related adverse events from combination immunotherapy in cancer patients: a comprehensive metaanalysis of randomized controlled trials. Int Immunopharmacol. 2018;63:292-8.

53. Larkin J, Chiarion-Sileni V, Gonzalez R, Grob JJ, Cowey CL, Lao CD, Schadendorf D, Dummer R, Smylie M, Rutkowski P, et al. Combined Nivolumab and Ipilimumab or monotherapy in untreated melanoma. $\mathrm{N}$ Engl J Med. 2015;373(1):23-34.

54. Ott PA, Hodi FS, Kaufman HL, Wigginton JM, Wolchok JD. Combination immunotherapy: a road map. J Immunother Cancer. 2017;5:16.

55. Xu C, Chen YP, Du XJ, Liu JQ, Huang CL, Chen L, Zhou GQ, Li WF, Mao YP, Hsu C, et al. Comparative safety of immune checkpoint inhibitors in cancer: systematic review and network meta-analysis. BMJ. 2018;363:k4226.

56. Sasidharan Nair V, Elkord E. Immune checkpoint inhibitors in cancer therapy: a focus on T-regulatory cells. Immunol Cell Biol. 2018;96(1):21-33.

57. Hellmann MD, Friedman CF, Wolchok JD. Combinatorial cancer immunotherapies. Adv Immunol. 2016;130:251.

58. Perez-Ruiz E, Etxeberria I, Rodriguez-Ruis ME, et al. Anti-CD137 and PD-1/PD-L1 antibodies en route toward clinical synergy[J]. Clin Cancer Res. 2017;23(18): 5326-8.

59. Chae YK, Arya A, lams W, Cruz MR, Chandra S, Choi J, Giles F. Current landscape and future of dual anti-CTLA4 and PD-1/PD-L1 blockade immunotherapy in cancer; lessons learned from clinical trials with melanoma and non-small cell lung cancer (NSCLC). J Immunother Cancer. 2018;6(1):39

60. Curran MA, Montalvo W, Yagita H, Allison JP. PD-1 and CTLA-4 combination blockade expands infiltrating $T$ cells and reduces regulatory $\mathrm{T}$ and myeloid cells within B16 melanoma tumors. Proc Natl Acad Sci U S A. 2010;107(9):4275-80.

61. Wolchok JD, Chiarion-Sileni V, Gonzalez R, Rutkowski P, Grob JJ, Cowey CL, Lao CD, Wagstaff J, Schadendorf D, Ferrucci PF. Overall survival with combined nivolumab and ipilimumab in advanced melanoma. N Engl J Med. 2017;377(14):1345.

62. Motzer RJ, Tannir NM, Mcdermott DF, et al. Nivolumab plus Ipilimumab versus Sunitinib in advanced renal-cell carcinoma[J]. N Engl J Med. 2018; 378(14):1277-90.

63. Hellmann MD, Ciuleanu TE, Pluzanski A, et al. Nivolumab plus Ipilimumab in lung cancer with a high tumor mutational burden. N Engl J Med. 2018; 378(22): 2093-104.

64. Lazennec G, Richmond A. Chemokines and chemokine receptors: new insights into cancer-related inflammation. Trends Mol Med. 2010;16(3):133-44.

65. Ji RR, Chasalow SD, Wang L, Hamid O, Schmidt H, Cogswell J, Alaparthy S, Berman D, Jure-Kunkel M, Siemers NO. An immune-active tumor microenvironment favors clinical response to ipilimumab. Cancer Immunol Immunother. 2012;61(7):1019-31.

66. Adams JL, Smothers J, Srinivasan R, Hoos A. Big opportunities for small molecules in immuno-oncology. Nat Rev Drug Discov. 2015;14(9):603-22.

67. Liu M, Wang X, Wang L, Ma X, Gong Z, Zhang S, Li Y. Targeting the IDO1 pathway in cancer: from bench to bedside. J Hematol Oncol. 2018;11(1):100.

68. Gangadhar TC, Hamid O, Smith DC, et al. Epacadostat plus pembrolizumab in patients with advanced melanoma and select solid tumors: updated phase 1 results from ECHO-202/KEYNOTE-037. Ann Oncol. 2016;27:1110PD. https://doi.org/10.1093/annonc/mdw379.06.

69. Perez RP, Riese MJ, Lewis KD, Saleh MN, Daud A, Berlin J, Lee JJ, Mukhopadhyay S, Zhou L, Serbest G, et al. Epacadostat plus nivolumab in patients with advanced solid tumors: preliminary phase $\mathrm{I} / \mathrm{II}$ results of $\mathrm{ECHO}$ 204. J Clin Oncol. 2017;35(15_suppl):3003.

70. Long GV, Dummer R, Hamid O, Gajewski T, Caglevic C, Dalle S, Arance A, Carlino MS, Grob J-J, Kim TM, et al. Epacadostat (E) plus pembrolizumab (P) versus pembrolizumab alone in patients (pts) with unresectable or metastatic melanoma: Results of the phase 3 ECHO-301/KEYNOTE-252 study. J Clin Oncol. 2018;36(15_suppl):108.

71. Zitvogel L, Galluzzi L, Smyth MJ, Kroemer G. Mechanism of action of conventional and targeted anticancer therapies: reinstating immunosurveillance. Immunity. 2013:39(1):74-88,

72. Liu L, Mayes PA, Eastman S, Shi H, Yadavilli S, Zhang T, Yang J, Seestaller-Wehr L, Zhang S-Y, Hopson C. The BRAF and MEK inhibitors dabrafenib and trametinib: effects on immune function and in combination with immunomodulatory antibodies targeting PD-1, PD-L1, and CTLA-4. Clin Cancer Res. 2015;21(7):1639-51.

73. Allen E, Jabouille A, Rivera LB, Lodewijckx I, Missiaen R, Steri V, Feyen K, Tawney J, Hanahan D, Michael IP. Combined antiangiogenic and anti-PD-L1 therapy stimulates tumor immunity through HEV formation. Sci Transl Med. 2017;9(385):eaak9679.

74. Campesato LF, Merghoub T. Antiangiogenic therapy and immune checkpoint blockade go hand in hand. Ann Transl Med. 2017;5(24):497. https://doi.org/10.21037/atm.2017.10.12.

75. Manegold C, Dingemans A-MC, Gray JE, Nakagawa K, Nicolson M, Peters S, Reck M, Wu Y-L, Brustugun OT, Crinò L. The potential of combined immunotherapy and antiangiogenesis for the synergistic treatment of advanced NSCLC. J Thorac Oncol. 2017:12(2):194-207.

76. Lee C-H, Makker V, Rasco DW, Taylor MH, Stepan DE, Shumaker RC, Schmidt EV, Guo M, Dutcus CE, Motzer RJ. Lenvatinib + pembrolizumab in patients with renal cell carcinoma: updated results. J Clin Oncol. 2018;36(15_suppl):4560.

77. Makker V, Rasco DW, Vogelzang NJ, Messing M, Brose MS, Cohn AL, Aghajanian C, Stepan DE, Dutcus CE, Schmidt EV, et al. Lenvatinib + pembrolizumab in patients with advanced endometrial cancer: updated results. J Clin Oncol. 2018;36(15_suppl):5596.

78. Golden EB, Frances D, Pellicciotta I, Demaria S, Helen Barcellos-Hoff M, Formenti SC. Radiation fosters dose-dependent and chemotherapy-induced immunogenic cell death. Oncoimmunology. 2014;3:e28518.

79. Formenti SC, Demaria S. Combining radiotherapy and cancer immunotherapy: a paradigm shift. J Natl Cancer Inst. 2013;105(4):256-65.

80. Demaria S, Golden EB, Formenti SC. Role of local radiation therapy in cancer immunotherapy. JAMA Oncol. 2015;1(9):1325-32.

81. De La Cruz-Merino L, Illescas Vacas A, Grueso López A, Barco Sánchez A Míguez Sánchez C, Cancer Immunotherapies Spanish Group Obo. Radiation for awakening the dormant immune system, a promising challenge to explore. Front Immunol. 2014;5:102. https://doi.org/10.3389/fimmu.2014.00102. 
82. Park SS, Dong H, Liu X, Harrington SM, Krco CJ, Grams MP, Mansfield AS, Furutani KM, Olivier KR, Kwon ED. PD-1 restrains radiotherapy-induced abscopal effect. Cancer Immunol Res. 2015;3(6):610-9.

83. Zeng J, See AP, Phallen J, Jackson CM, Belcaid Z, Ruzevick J, Durham N, Meyer C, Harris TJ, Albesiano E. Anti-PD-1 blockade and stereotactic radiation produce long-term survival in mice with intracranial gliomas. Int J Radiat Oncol Biol Phys. 2013;86(2):343-9.

84. Antonia SJ, Villegas A, Daniel D, Vicente D, Murakami S, Hui R, Yokoi T, Chiappori A, Lee KH, de Wit M. Durvalumab after chemoradiotherapy in stage III non-small-cell lung cancer. N Engl J Med. 2017;377(20):1919-29.

85. Luke JJ, Lemons JM, Karrison TG, Pitroda SP, Melotek JM, Zha Y, Al-Hallaq HA, Arina A, Khodarev NN, Janisch L. Safety and clinical activity of pembrolizumab and multisite stereotactic body radiotherapy in patients with advanced solid tumors. J Clin Oncol. 2018;36(16):1611-8.

86. Bracci L, Schiavoni G, Sistigu A, Belardelli F. Immune-based mechanisms of cytotoxic chemotherapy: implications for the design of novel and rationale-based combined treatments against cancer. Cell Death Differ. 2014;21(1):15-25.

87. Zitvogel L, Kepp O, Kroemer G. Immune parameters affecting the efficacy of chemotherapeutic regimens. Nat Rev Clin Oncol. 2011;8(3):151-60.

88. Apetoh L, Mignot G, Panaretakis T, Kroemer G, Zitvogel L. Immunogenicity of anthracyclines: moving towards more personalized medicine. Trends $\mathrm{Mol}$ Med. 2008;14(4):141-51.

89. Sharma MD, Hou D-Y, Baban B, Koni PA, He Y, Chandler PR, Blazar BR, Mellor AL, Munn DH. Reprogrammed Foxp3+ regulatory T cells provide essential help to support cross-presentation and CD8+ T cell priming in naive mice. Immunity. 2010;33(6):942-54

90. Shevchenko I, Karakhanova S, Soltek S, Link J, Bayry J, Werner J, Umansky V, Bazhin AV. Low-dose gemcitabine depletes regulatory $T$ cells and improves survival in the orthotopic Panc02 model of pancreatic cancer. Int J Cancer. 2013;133(1):98-107.

91. Sevko A, Michels T, Vrohlings M, Umansky L, Beckhove P, Kato M, Shurin GV, Shurin MR, Umansky V. Antitumor effect of paclitaxel is mediated by inhibition of myeloid-derived suppressor cells and chronic inflammation in the spontaneous melanoma model. J Immunol. 2013;190:2464-71.

92. Pfirschke C, Engblom C, Rickelt S, Cortez-Retamozo V, Garris C, Pucci F, Yamazaki T, Poirier-Colame V, Newton A, Redouane Y. Immunogenic chemotherapy sensitizes tumors to checkpoint blockade therapy. Immunity. 2016:44(2):343-54

93. Borghaei H, Langer CJ, Gadgeel S, Papadimitrakopoulou VA, Patnaik A, Powell SF, Gentzler RD, Martins RG, Stevenson JP, Jalal SI, et al. 24-month overall survival from KEYNOTE-021 cohort G: Pemetrexed and Carboplatin with or without Pembrolizumab as first-line therapy for advanced nonsquamous non-small cell Lung cancer. J Thorac Oncol. 2019;14(1):124-9.

94. Gandhi L, Rodríguez-Abreu D, Gadgeel S, Esteban E, Felip E, De Angelis F, Domine M, Clingan P, Hochmair MJ, Powell SF. Pembrolizumab plus chemotherapy in metastatic non-small-cell lung cancer. N Engl J Med. 2018; 378(22):2078-92.

95. Fuchs CS, Doi T, Jang RW, et al. Safety and efficacy of pembrolizumab monotherapy in patients with previously treated advanced gastric and gastroesophageal junction cancer: phase 2 clinical keynote-059 trial. JAMA Oncol. 2018;4(5):e180013.

96. Doi T, Piha-Paul SA, Jalal SI, Saraf S, Lunceford J, Koshiji M, Bennouna J. Safety and antitumor activity of the anti-programmed death-1 antibody pembrolizumab in patients with advanced esophageal carcinoma. J Clin Oncol. 2018;36(1):61-7.

97. Shah MA, Kojima T, Enzinger PC, Hochhauser D, Raimbourg J, Hollebecque A, Lordick F, Kim S-B, Tajika M, Kim HT. Pembrolizumab for patients with previously treated metastatic adenocarcinoma or squamous cell carcinoma of the esophagus: phase 2 KEYNOTE-180 study. Am Soc Clin Oncol. 2018;36: 4049. https://doi.org/10.1200/JCO.2018.36.15_suppl.4049.

98. Janjigian YY, Bendell J, Calvo E, Kim JW, Ascierto PA, Sharma P, Ott PA, Peltola K, Jaeger D, Evans J. CheckMate-032 study: efficacy and safety of nivolumab and nivolumab plus ipilimumab in patients with metastatic esophagogastric cancer. J Clin Oncol. 2018;36(28):2836-44.

99. Bang Y-J, Muro K, Fuchs CS, Golan T, Geva R, Hara H, Jalal SI, Borg C, Doi T, Wainberg ZA, et al. KEYNOTE-059 cohort 2: safety and efficacy of pembrolizumab (pembro) plus 5-fluorouracil (5-FU) and cisplatin for first-line (1L) treatment of advanced gastric cancer. J Clin Oncol. 2017;35(15_suppl):4012.

100. Takahari D, Shoji H, Hara H, Esaki T, Machida N, Nagashima K, Aoki K, Honda K, Miyamoto T, Boku N. Preliminary result of phase $1 / 2$ study of ramucirumab plus nivolumab in patients with previously treated advanced gastric adenocarcinoma (NivoRam study). Am Soc Clin Oncol. 2018;36:4047.

101. Klempner SJ, Bendell J, Meucci Villaflor V, Tenner L, Stein S, Sirard CA, Kagey M, Newman W, Schlienger K, Strickler J. 660PSafety and efficacy of a DKK1 inhibitor (DKN-01) in combination with pembrolizumab (P) in patients (Pts) with advanced gastroesophageal (GE) malignancies. Ann Oncol. 2018;29:mdy282.044. https://doi. org/10.1093/annonc/mdy282.044

102. Kang Y-K, Yamaguchi K, Hara H, Fumita S, Azuma M, Boku N, Chen L-T, Kato K, Chung HC, Minashi K, et al. 671PInterim safety and clinical activity of nivolumab (Nivo) in combination with S-1/capecitabine plus oxaliplatin in patients (pts) with previously untreated unresectable advanced or recurrent gastric/gastroesophageal junction (G/GEJ) cancer: part 1 study of ATTRACTION-04 (ONO-4538-37). Ann Oncol. 2017;28:mdx369.055. https://doi. org/10.1093/annonc/mdx369.055.

103. Catenacci DVT, Lee K-W, Yen J, Odegaard J, Franovic A, Baughman J, Muth J, Wynter-Horton A, Wu T, Wigginton J, et al. 662PBiomarker-guided enrichment of the antitumor activity of margetuximab $(M)$ plus pembrolizumab $(P)$ in patients with advanced HER2+ gastric adenocarcinoma (GEA). Ann Oncol. 2018;29:mdy282.046. https://doi.org/10.1093/annonc/mdy282.046.

104. Shah MA, Metges J-P, Cunningham D, Shiu K-K, Wyrwicz L, Thai D, Brachmann C, Bhargava P, Catenacci DVT, Wainberg ZA. A phase II, openlabel, randomized study to evaluate the efficacy and safety of andecaliximab combined with nivolumab versus nivolumab alone in subjects with unresectable or recurrent gastric or gastroesophageal junction adenocarcinoma. J Clin Oncol. 2019;37(4_suppl):75.

105. Janjigian YY, Chou JF, Simmons M, Momtaz P, Sanchez-Vega F, Shcherba M, Ku GY, Won E, Chong CR, Gerdes H, et al. First-line pembrolizumab (P), trastuzumab ( $\mathrm{T})$, capecitabine $(\mathrm{C})$ and oxaliplatin (O) in HER2-positive metastatic esophagogastric adenocarcinoma (mEGA). J Clin Oncol. 2019; 37(4_suppl):62.

106. Catenacci DVT, Lim KH, Uronis HE, Kang Y-K, Ng MCH, Gold PJ, Enzinger PC, Lee KW, Lacy J, Park SH, et al. Antitumor activity of margetuximab (M) plus pembrolizumab (P) in patients (pts) with advanced HER2+ $(\mathrm{IHC} 3+)$ gastric carcinoma (GC). J Clin Oncol. 2019;37(4_suppl):65.

107. Hara H, Shoji H, Takahari D, Esaki T, Machida N, Nagashima K, Aoki K, Honda K, Miyamoto T, Boku N, et al. Phase I/II study of ramucirumab plus nivolumab in patients in second-line treatment for advanced gastric adenocarcinoma (NivoRam study). J Clin Oncol. 2019;37(4_suppl):129.

108. Network TCGA. Comprehensive molecular characterization of gastric adenocarcinoma. Nature. 2014;513(7517):202-9.

109. Le DT, Uram JN, Wang $H$, Bartlett BR, Kemberling $H$, Eyring AD, Skora AD, Luber BS, Azad NS, Laheru D, et al. PD-1 blockade in tumors with mismatchrepair deficiency. N Engl J Med. 2015;372(26):2509-20.

110. Satoh T, Chen LT, Kang YK, Chao Y, Kato K, Chung HC, Chen JS, Muro K, Kang WK, Yoshikawa T, et al. 617PDA phase III study of nivolumab (nivo) in previously treated advanced gastric or gastric esophageal junction (G/GEJ) cancer (ATTRACTION-2): Two-years update data. Ann Oncol. 2018;29(suppl_ 8):mdy282.002.

111. Petrylak DP, Arkenau H-T, Perez-Gracia JL, Krebs M, Santana-Davila R, Yang J, Rege J, Mi G, Ferry D, Herbst RS. A multicohort phase I study of ramucirumab (R) plus pembrolizumab (P): interim safety and clinical activity in patients with urothelial carcinoma. J Clin Oncol. 2017;35(6_suppl):349.

112. Kato K, Shah MA, Enzinger PC, Bennouna J, Shen L, Adenis A, Sun JM, Cho BC, Ozguroglu M, Kojima T, et al. 785TiPPhase III KEYNOTE-590 study of chemotherapy + pembrolizumab versus chemotherapy + placebo as firstline therapy for patients (Pts) with advanced esophageal or esophagogastric junction (E/EGJ) cancer. Ann Oncol. 2018;29(suppl_8):mdy282.168.

113. El-Khoueiry AB, Sangro B, Yau T, Crocenzi TS, Kudo M, Hsu C, Kim TY, Choo SP, Trojan J, Rd WT. Nivolumab in patients with advanced hepatocellular carcinoma (CheckMate 040): an open-label, non-comparative, phase 1/2 dose escalation and expansion trial. Lancet (Lond. Engl.). 2017;389(10088):2492.

114. Zhu AX, Finn RS, Cattan S, Edeline J, Ogasawara S, Palmer DH, Verslype C, Zagonel V, Rosmorduc O, Vogel A, et al. KEYNOTE-224: pembrolizumab in patients with advanced hepatocellular carcinoma previously treated with sorafenib. J Clin Oncol. 2018;36(4_suppl):209.

115. Sangro B, Park J-W, Cruz CMD, Anderson J, Lang L, Neely J, Shaw JW, Cheng A-L. A randomized, multicenter, phase 3 study of nivolumab vs sorafenib as first-line treatment in patients (pts) with advanced hepatocellular carcinoma (HCC): CheckMate-459. J Clin Oncol. 2016;34(15_suppl):TPS4147.

116. Gu P, Park J, Zhong J, Guo S, Hickey R, Aaltonen E, Horn J, Du KL, Shanbhogue K, Megibow A, et al. Initial experience of combination 
nivolumab and local-regional treatment in patients with advanced hepatocellular carcinoma (HCC). J Clin Oncol. 2018;36(15_suppl):e16149.

117. Ikeda M, Sung MW, Kudo M, Kobayashi M, Baron AD, Finn RS, Kaneko S, Zhu AX, Kubota T, Kraljevic S, et al. A phase 1b trial of lenvatinib (LEN) plus pembrolizumab (PEM) in patients (pts) with unresectable hepatocellular carcinoma (uHCC). J Clin Oncol. 2018;36(15_suppl):4076.

118. Pishvaian MJ, Lee MS, Ryoo BY, Stein S, Lee KH, Verret W, Spahn J, Shao H, Liu B, lizuka K, et al. LBA26Updated safety and clinical activity results from a phase Ib study of atezolizumab + bevacizumab in hepatocellular carcinoma (HCC). Ann Oncol. 2018;29(suppl_8):mdy424.028.

119. Kelley RK, Abou-Alfa GK, Bendell JC, Kim T-Y, Borad MJ, Yong W-P, Morse M, Kang $Y$-K, Rebelatto M, Makowsky $M$, et al. Phase I/II study of durvalumab and tremelimumab in patients with unresectable hepatocellular carcinoma (HCC): Phase I safety and efficacy analyses. J Clin Oncol. 2017;35(15_suppl):4073.

120. Ikeda M, Ueno M, Morizane C, Kobayashi S, Ohno I, Kondo S, Okano N, Kimura K, Asada S, Namba Y, et al. A multicenter, open-label, phase I study of nivolumab alone or in combination with gemcitabine plus cisplatin in patients with unresectable or recurrent biliary tract cancer. J Clin Oncol. 2019;37(4_suppl):306.

121. Lin J, Shi W, Zhao S, Hu J, Hou Z, Yao M, Chrin G, Pan J, Hu K, Zhao L, et al. Lenvatinib plus checkpoint inhibitors in patients (pts) with advanced intrahepatic cholangiocarcinoma (ICC): Preliminary data and correlation with next-generation sequencing. J Clin Oncol. 2018;36(4_suppl):500.

122. loka T, Ueno M, Oh D-Y, Fujiwara Y, Chen J-S, Doki Y, Mizuno N, Park K, Asagi A, Hayama M, et al. Evaluation of safety and tolerability of durvalumab (D) with or without tremelimumab (T) in patients (pts) with biliary tract cancer (BTC). J Clin Oncol. 2019;37(4_suppl):387.

123. Floudas CS, Xie C, Brar G, Morelli MP, Fioravanti S, Walker M, Mabry-Hrones D, Wood BJ, Levy EB, Krishnasamy VP, et al. Combined immune checkpoint inhibition $(\mathrm{ICl})$ with tremelimumab and durvalumab in patients with advanced hepatocellular carcinoma (HCC) or biliary tract carcinomas (BTC). J Clin Oncol. 2019:37(4_suppl):336.

124. Hidalgo M, Park JO, Ramirez R, Peled A, Vainstein Haras A, Rosenfeld O, Lustig TM, Sorani E, Bohana Kashtan O, Schlienger K, et al. 1133PDA phase Ila trial to assess the safety and efficacy of BL-8040 and pembrolizumab in patients with metastatic pancreatic adenocarcinoma (PDAC). Ann Oncol. 2018;29:mdy288.006. https://doi.org/10.1093/annonc/mdy288.006.

125. Wainberg ZA, Hochster HS, Kim EJ-H, George B, Kalyan A, Chiorean EG, Waterhouse DM, Gutierrez M, Parikh AR, Jain R, et al. Phase I study of nivolumab (Nivo) + nab-paclitaxel (nab-P) + gemcitabine (Gem) in advanced pancreatic cancer (APC). J Clin Oncol. 2019;37(4_suppl):298.

126. Brar G, Xie C, Floudas CS, Morelli MP, Fioravanti S, Walker M, Mabry-Hrones D, Jones JC, Greten TF. Immune checkpoint inhibition (ICI) in combination with SBRT in patients with advanced pancreatic adenocarcinoma (aPDAC). J Clin Oncol. 2019;37(4_suppl):192.

127. Ducreux MP, Cheng AL, Qin S, Zhu AX, Ikeda M, Kim TY, Xu DZ, Verret W, Liu J, Finn RS, et al. 782TiPAtezolizumab + bevacizumab vs sorafenib in locally advanced or metastatic hepatocellular carcinoma: The randomised phase III study IMbrave150. Ann Oncol. 2018;29(suppl_8):mdy282.165.

128. Nakamura H, Arai Y, Totoki Y, Shirota T, Elzawahry A, Kato M, Hama N, Hosoda F, Urushidate T, Ohashi S. Genomic spectra of biliary tract cancer. Nat Genet. 2015;47(9):1003.

129. Bang YJ, Doi T, Braud FD, Piha-Paul S, Hollebecque A, Razak ARA, Lin CC, Ott PA, He AR, Yuan SS. 525 Safety and efficacy of pembrolizumab (MK-3475) in patients (pts) with advanced biliary tract cancer: Interim results of KEYNOTE028. Eur J Cancer. 2015;51:S112.

130. Thind K, Padrnos LJ, Ramanathan RK, Borad MJ. Immunotherapy in pancreatic cancer treatment: a new frontier. Therap Adv Gastroenterol. 2017;10(1):168-94.

131. Protti MP, De Monte L. Immune infiltrates as predictive markers of survival in pancreatic cancer patients. Front Physiol. 2013;4:210.

132. O'Neil BH, Wallmark J, Lorente D, Elez E, Raimbourg J, Gomez-Roca C, Ejadi S, Piha-Paul SA, Moss RA, Siu LL. Pembrolizumab (MK-3475) for patients (pts) with advanced colorectal carcinoma (CRC): Preliminary results from KEYNOTE-028. Eur J Cancer. 2015;51:S103.

133. Overman MJ, McDermott R, Leach JL, Lonardi S, Lenz HJ, Morse MA, Desai J, Hill A, Axelson M, Moss RA, et al. Nivolumab in patients with metastatic DNA mismatch repair-deficient or microsatellite instability-high colorectal cancer (CheckMate 142): an open-label, multicentre, phase 2 study. Lancet Oncol. 2017;18(9):1182-91.
134. Overman MJ, Lonardi S, Wong KYM, Lenz HJ, Gelsomino F, Aglietta M, Morse MA, Van Cutsem E, McDermott R, Hill A, et al. Durable clinical benefit with nivolumab plus ipilimumab in DNA mismatch repair-deficient/ microsatellite instability-high metastatic colorectal cancer. J Clin Oncol. 2018;36(8):773-9.

135. Bendell JC, Powderly JD, Lieu CH, Eckhardt SG, Hurwitz H, Hochster HS, Murphy JE, Funke RP, Rossi C, Wallin J, et al. Safety and efficacy of MPDL3280A (anti-PDL1) in combination with bevacizumab (bev) and/or FOLFOX in patients (pts) with metastatic colorectal cancer (mCRC). J Clin Oncol. 2015;33(3_suppl):704.

136. Bendell JC, Bang Y-J, Chee CE, Ryan DP, McRee AJ, Chow LQ, Desai J, Wongchenko M, Yan Y, Pitcher B, et al. A phase Ib study of safety and clinical activity of atezolizumab (A) and cobimetinib (C) in patients (pts) with metastatic colorectal cancer (mCRC). J Clin Oncol. 2018;36(4_suppl):560.

137. Segal NH, Kemeny NE, Cercek A, Reidy DL, Raasch PJ, Warren P, Hrabovsky AE, Campbell N, Shia J, Goodman KA, et al. Non-randomized phase II study to assess the efficacy of pembrolizumab (Pem) plus radiotherapy (RT) or ablation in mismatch repair proficient (pMMR) metastatic colorectal cancer (mCRC) patients. J Clin Oncol. 2016;34(15_suppl):3539.

138. Yarchoan M, Ferguson AK, Durham JN, Rozich N, Rodriguez C, Huang C-Y, Browner IS, Jesus-Acosta AD, Le DT, Laheru D, et al. A phase II study of GVAX colon vaccine with cyclophosphamide and pembrolizumab in patients with mismatch repair-proficient (MMR-p) advanced colorectal cancer. J Clin Oncol. 2019;37(4_suppl):563.

139. Chen EX, Jonker DJ, Kennecke HF, Berry SR, Couture F, Ahmad CE, Goffin JR, Kavan P, Harb M, Colwell B, et al. CCTG CO.26 trial: a phase II randomized study of durvalumab (D) plus tremelimumab (T) and best supportive care (BSC) versus BSC alone in patients (pts) with advanced refractory colorectal carcinoma (rCRC). J Clin Oncol. 2019;37(4_suppl):481.

140. Ebert PJ, Cheung J, Yang Y, McNamara E, Hong R, Moskalenko M, Gould SE, Maecker H, Irving BA, Kim JM. MAP kinase inhibition promotes T cell and anti-tumor activity in combination with PD-L1 checkpoint blockade. Immunity. 2016;44(3):609-21.
Ready to submit your research? Choose BMC and benefit from:
- fast, convenient online submission
- thorough peer review by experienced researchers in your field
- rapid publication on acceptance
- support for research data, including large and complex data types
- gold Open Access which fosters wider collaboration and increased citations
- maximum visibility for your research: over $100 \mathrm{M}$ website views per year
At BMC, research is always in progress. 